3D effects of edge magnet i c fi el d

conf $i$ gur at $i$ on on di vert or /scrape- of $f$ I ayer transport and opti mzation possibilities for a f ut ure react or

\begin{tabular}{|l|l|}
\hline $\begin{array}{l}\text { jour nal or } \\
\text { publ i cat i on } \mathrm{ti} \text { t l e }\end{array}$ & Nucl ear Fusi on \\
\hline vol une & 55 \\
\hline nunber & 10 \\
\hline page $\mathrm{r}$ ange & 104021 \\
\hline year & 2015- 09-29 \\
\hline URL & ht t p: //hdl . handl e. net /10655/00012558 \\
\hline
\end{tabular}




\section{D effects of edge magnetic field configuration on divertor/SOL transport and optimization possibilities for a future reactor}

M. Kobayashi ${ }^{1}$, Y. Xu ${ }^{2}$, K. Ida ${ }^{1}$, Y. Corre ${ }^{3}$, Y. Feng ${ }^{4}$, O. Schmitz ${ }^{5}$, H. Frerichs ${ }^{5}$, F.L. Tabares ${ }^{6}$, T.E. Evans ${ }^{7}$, J.W. Coenen ${ }^{8}$, Y. Liang ${ }^{8}$, A. Bader ${ }^{5}$, K. Itoh ${ }^{1}$, H. Yamada ${ }^{1}$, Ph. Ghendrih ${ }^{3}$, G. Ciraolo $^{3}$, D. Tafalla ${ }^{6}$, A. Lopez-Fraguas ${ }^{6}$, H.Y. Guo ${ }^{7}{ }^{9}$, Z.Y. Cui ${ }^{2}$, D. Reiter ${ }^{8}$, N. Asakura ${ }^{10}$, U. Wenzel $^{4}$, S. Morita ${ }^{1}$, N. Ohno ${ }^{11}$, B.J. Peterson ${ }^{1}$, and S. Masuzaki ${ }^{1}$

${ }^{1}$ National Institute for Fusion Science, 322-6 Oroshi-cho, Toki 509-5292, Japan

${ }^{2}$ Southwestern Institute of Physics, P. O. Box 432, Chengdu 610041, China

${ }^{3}$ IRFM, CEA Cadarache ST Paul Lez Durance, France

${ }^{4}$ Max-Planck-Institute fuer Plasmaphysik, D-17491 Greifswald, Germany

${ }^{5}$ University of Wisconsin - Madison, WI, USA

${ }^{6}$ Laboratorio Nacional de Fusion. Ciemat, Madrid, Spain

${ }^{7}$ General Atomics, P.O. Box 85608, San Diego, California 92186-5608, USA

${ }^{8}$ Forschungszentrum Jülich GmbH Institut für Energie- und Klimaforschung - Plasmaphysik, Jülich, Germany

${ }^{9}$ Institute of Plasma Physics, CAS, Hefei, China

10 Japan Atomic Energy Agency, Rokkasho, Aomori 039-3212, Japan

${ }^{11}$ Nagoya University, Nagoya 464-8603, Japan

PACS; 52.55.Hc, 52.55.Fa, 52.55.Rk, 52.25.Fi

\section{Abstract}

This paper assesses the 3D effects of edge magnetic field structure on divertor/SOL transport, based on inter-machine comparison of experimental data and on the recent progress of 3D edge transport simulation. The 3D effects are elucidated as a consequence of competition between transports parallel $(/ /)$ and perpendicular $(\perp)$ to magnetic field, in open field lines cut by divertor plates, or in magnetic islands. The competition has strong impacts on divertor functions, such as determination of the divertor density regime, impurity screening and detachment control. The effects of magnetic perturbation on the edge electric field and turbulent transport are also discussed. Parameterization to measure the 3D effects on the edge transport is attempted for the individual divertor functions. Based on the suggested key parameters, an operation domain of the 3D divertor configuration is discussed for future devices. 


\section{Introduction}

Since the concept of a divertor was first proposed in around 1950's [1,2,3,4] in order to avoid intimate contact of the plasma confinement region with material surfaces, numerous efforts have been dedicated to the understanding of the divertor plasma $[5,6,7,8,9,10,11]$. The optimization of the tokamak divertor strongly depends on the divertor geometry, i.e. the geometry of flux surfaces and shape of divertor/baffle plates and their relative distance from each other. Divertor optimization has to be considered taking into account various issues, the peak heat load at divertor plates, the operating range of detached plasmas, particle control with efficient pumping, He exhaust capability and impurity transport. In helical devices, the situation might be even more complex compared to the 2D axi-symmetric tokamaks because of the 3D non axi-symmetric magnetic field and divertor/first wall structure $[12,13,14,15,16,17,18,19]$. Complexity also appears in tokamaks with the application of symmetry breaking magnetic perturbation (MP) fields aimed at edge transport control [ $20,21,22,23,24,25,26,27$ ] or at ELM mitigation or suppression $[25,28,29,30,31,32,33,34,35,36,37,38,39,40,41]$. In tokamaks, the non-axisymmetric magnetic field structure appears also due to intrinsic 3D fields (error fields, test blanket modules), due to 3D effects such as neoclassical tearing modes, non-axisymmetric particle, energy and momentum sources, and 3D plasma facing component geometry. An understanding of the 3D effects is rooted, therefore, in common or complementary issues between tokamaks and helical devices, and is one of the most demanding topics for divertor optimization toward a future reactor, exploring advanced magnetic configurations $[14,42,43,44,45]$.

In recent years, there has been significant progress in the 3D numerical simulation codes, EMC3-EIRENE [46,47], TOKAM-3X, -3D [48,49,50], E3D [51], FINDIF [52] etc., which thus enable us to analyse the experimental data accumulated in various devices $[53,54,55,56,23,57,58,59,60]$. The 3D effects have been intensely investigated so far in each device and significant progress has been made in the understanding of transport features $[20,23,50,55,61,62,63,64,65,66]$. This paper aims at obtaining an overview of the 3D effects on divertor/SOL transport by extending the operating parameter range over different devices by collecting data from various tokamaks and helical devices. The collected data of 
experiments as well as numerical simulation are compared according to a parameterization to measure the $3 \mathrm{D}$ effects of magnetic field structure. The analysis especially focuses on the impacts on the divertor density regime, impurity transport, detachment stability, and edge electric field formation and turbulent transport. Based on the analysis, possible optimization of the 3D divertor configuration for future devices is discussed. The rest of the paper is organized as follows. In the next section, we define the 3D effects that are discussed in this article. In Section 3 to 6, we present the impacts of 3D magnetic field configuration on the divertor density regime, impurity transport, detachment stability and edge electric field/turbulent transport, respectively. Section 7 summarizes the paper.

\section{The definition of 3D effects}

There is a variety of magnetic configurations for divertors depending on the concept of optimizations. In this paper, in order to define the 3D effects, which can be applied for all the devices investigated here, we make categorization of the divertor configurations as follows:

\section{Type I: Limiter + MP}

The MP is applied to the limiter configuration, which produce the edge stochastic layers and the laminar region close to the divertor plates as shown in Fig.1 (a). We introduce coordinates $r$ and $\theta$, which represent radial and poloidal directions, respectively.

Stochastization of the magnetic field due to destruction of the magnetic surface was predicted already at the early stage of fusion plasma research [67,68]. Later, such deformation of the flux surface has been confirmed also in experiments $[27,69,70,71]$. The transport is considered to be affected in the stochastic layer due to radial projection of the field lines caused by the magnetic field braiding [72,73,74].

In this type, the transport between the last closed flux surface, LCFS, and the divertor plates is mainly carried out by radial projection of the //-transport along the stochastic field lines, $\Gamma_{/ /}$, and the radial component of transport perpendicular $(\perp)$ to the field lines, $\Gamma_{\perp r}$. Here, the flux $\Gamma_{/ /}$and $\Gamma_{\perp}$ represent either particle, momentum, or energy. TEXTOR-DED and Tore Supra belong to this category. 
Type II: Island divertor

In this case, the edge islands are cut by divertor plates as shown in Fig.1 (b). Each island forms a scrape-off layer (SOL), which is well separated each other. In this type, the transport between the LCFS and the divertor plates is carried out by radial projection of the //-transport along the island field lines, $\Gamma_{/ /}$, and the radial component of transport perpendicular to the field lines, $\Gamma_{\perp r}$. W7-AS, W7-X and HSX correspond to this type. The configuration of TJ-II referred in this paper has an island-type configuration [75], and thus belongs to this type.

Type III: X-point poloidal divertor + MP, helical divertor

This type appears when MP is applied to the X-point poloidal divertor configuration or naturally in the helical divertor configuration. An overlap of the islands induced by the MP or of islands naturally occurring due to the side band spectrum of magnetic field component of helical coils introduces the stochastic layer. Near the divertor plates, divertor legs are split connecting to different locations on the divertor plates. The transport between the LCFS (perturbed separatrix) and the divertor plates is mainly carried out by $\Gamma_{/ /}$and a poloidal component of transport perpendicular to the field lines, $\Gamma_{\perp \theta}$. The radial component of transport perpendicular to the field lines, $\Gamma_{\perp r}$, also plays a role in several aspects, e.g. to determine the SOL width and a deposition profiles on the divertor, to flush out impurities etc.. DIII-D, EAST, ITER and LHD correspond to this type.

Particularly in the present study, in order to obtain a simple picture, the 3D effects are defined as follows. The 3D effects emerge when the //-transport starts to compete with the $\perp$-transport to deliver particle, momentum or energy from upstream (i.e., around the LCFS, or around the midplane in X-point divertor) to the downstream (i.e., divertor region). This situation occurs in open field lines of the stochastic layer or also of magnetic islands, as shown in Fig.1. In this paper, we restrict ourselves to the prescribed magnetic field structure for simplicity of the analysis, and therefore the dynamical or static change of the magnetic field structure due to plasma response is beyond the scope of this paper. Here we consider the case, for which the 3D effects appear, 


$$
\Gamma_{/ /} \Theta \sim \Gamma_{\perp r} \text { or } \Gamma_{\perp \theta},
$$

where $\Theta$ is a field line pitch [62], and can be written as $\Theta \equiv B_{r} / B_{t}$ or $B_{\theta} / B_{t}$, depending on the flux component on the right hand side, with $B_{r}, B_{\theta}$ and $B_{t}$ representing a radial, poloidal and toroidal component of the magnetic field, respectively. In other words, the 3D effects appear when the //-transport time to travel along the open field lines or to circulate inside island from the upstream to the downstream, becomes comparable to the $\perp$ - transport time that is needed to cross the field lines from the upstream to the downstream.

For typical SOL plasma conditions, e.g. $T_{e}=10 \sim 100 \mathrm{eV}, n_{e}=10^{19} \sim 10^{20} \mathrm{~m}^{-3}$, and if we assume that a perpendicular scale length is an order of $1 \sim 10 \mathrm{~cm}$ and a parallel scale length is an order of $10 \mathrm{~m}$ (i.e. $\nabla_{/ /} \sim 0.1 \mathrm{~m}^{-1}$ ), then the ratio $\Gamma_{/ /} / \Gamma_{\perp r, \theta}$ is estimated at more than $10^{2}$. In the SOL of $2 \mathrm{D}$ axi-symmetric X-point divertor tokamaks, in which case we take $B_{\theta}$ in Eq.(1) since the flux from the upstream to the divertor is directed mainly in poloidal direction, the ratio typically becomes $\frac{B_{\theta}}{B_{t}} \sim 0.1$. In this case, we have the situation such as $\Gamma_{/ /}\left(\frac{B_{\theta}}{B_{t}}\right)>>\Gamma_{\perp \theta}$, thus the //-transport dominates over $\perp$ transport. This argument should be distinguished from the hypothesis that the radial SOL width in the 2D axi-symmetric divertor is determined by balancing a $\perp$ (radial) transport and a // transport.

On the other hand, in the radial direction in the type I to III shown in Fig.1, the ratio $\frac{B_{r}}{B_{t}}=10^{-4} \sim 10^{-3}$, and thus it can be realized that $\Gamma_{/ /}\left(\frac{B_{r}}{B_{t}}\right) \sim \Gamma_{\perp r}$. In such case, the $\perp$-transport contributes substantially to deliver particles, momentum and energy. The parameter domain in which this effect becomes significant is elucidated in Fig.8 in ref.[76].

For a vector quantity, such as momentum transport, the direction of the flow or of the field line connection to the divertor plates is also important. When the flow fields have (toroidally) opposite streaming directions and thus interact with each other due to the proximity of the counter connecting field lines, they lose the //-momentum via dissipation caused by $\perp$-viscosity. Figure 2 is a schematic of the flow direction along flux tubes connected to divertor plates, where $\lambda_{m}$ represents the spatial separation between the counter streaming flows. The loss of //-momentum can occur in the island divertor [76] or in the stochastic layer [63], in which case $\lambda_{m}$ can be small compared to perpendicular 
characteristic length of momentum transport. In the 2D axi-symmetric X-point divertor, the separation of counter directional flux tubes, i.e., inner and outer divertor legs, are usually large, and thus the momentum loss effect is considered to be negligible.

The enhancement of $\perp$-transport also occurs in the situation where short and long connection length flux tubes are touching each other and they are strongly stretched, bended and folded due to the magnetic shear and a flux conservation, as shown in Fig.3. When the density is high enough in these configurations, the exchange of physical quantities such as particle and energy between the different flux tubes is considered to be enhanced due to the enlarged interface between them.

It is noted that, in closed nested flux surfaces the //-transport uniformly distributes plasma parameters along the flux surfaces due to the fast transport. In such cases, the //-transport does not contribute to "net" transport from the LCFS to divertor plates (or "net" radial direction), even with non-axisymmetric deformation of the magnetic flux surfaces. Therefore, no 3D effects are expected in terms of the definition in the present analysis.

Certainly, there are other 3D effects such as neoclassical transport in symmetry breaking fields $[77,78]$, resulting in enhanced ripple losses, which can affect both cross-field transport [79] and momentum damping, e.g. NTV [80,81]. Also an important issue is a modification of heat and particle flux distributions on divertor plates due to a strike line splitting $[36,55,57,82]$. These effects can affect, for example, impurity transport through changes of impurity source distribution due to the strike line splitting, and also due to the modified electric field caused by the neoclassical effects. While it is recognized that these topics are also important, the effects are not covered in this paper.

The important parameters used to obtain the parameterization of the 3D effects are listed in Table 1 for various devices, which contributed to the present analysis. The data were obtained in the references indicated for each device. Some of them were also provided through the private communications between the authors.

In the following sections, we analyse how these 3D effects affect the experimental observations and then discuss possible impacts on divertor functions.

\section{3D effects on divertor density regime}


The divertor density regime, which characterizes the relation between upstream (LCFS or midplane) and downstream (divertor) plasma parameters, is an important feature of the divertor operation, through which the core plasma performance and the divertor operation is correlated. The divertor density regime in the 2D axi-symmetric X-point divertor configuration is known for strong recycling enhancement in the divertor region, which is sustained with the sufficient upstream plasma pressure and energy input needed for the ionization process and with effective neutral confinement in the divertor region [83]. In this case, the divertor (downstream) density and temperature are strongly coupled to the upstream (LCFS or midplane) density. For example, the nonlinear relations $n_{\text {down }} \propto n_{\text {up }}{ }^{3}, T_{\text {down }} \propto n_{\text {up }}{ }^{-2}$, are derived from the pressure conservation along flux tubes between the upstream and the downstream [83,84]. Such a density regime is called the high-recycling regime or conduction limited regime [83] and the relation has been confirmed in experiments [85, 86, 87, 88, 89, 90, 91]. In the analysis in this section we restrict ourselves to the attached phase with most of the ionization occurring at the divertor plate. The observations presented in this section are in the relatively high collisional cases, i.e. the condition with SOL collisionality, $v_{S O L}{ }^{*}=L_{C} / \lambda_{e e}$, more than 10 , where $L_{C}$ and $\lambda_{e e}$ are field line connection length and electron mean-free path, respectively. In this case, one expects the high recycling regime in the $2 \mathrm{D}$ axi-symmetric tokamaks. It is also noted that all experiments presented here are in the range $T_{\text {down }} \geq 10 \mathrm{eV}$ before the detachment transition, as shown later in Fig.4. On the other hand, significant momentum loss via plasma neutral interaction is expected at $T_{\text {down }} \leq 5[92,93,94]$. Therefore, the effect of neutrals on the momentum transport is considered to be small in the present cases.

\subsection{Absence of high recycling regime due to loss of //-momentum}

In the 3D divertor configurations such as helical devices, W7-AS [95,96], LHD [97,98] and tokamaks with an MP, TEXTOR-DED [99], it has been sometimes found that the dependency becomes modest, i.e., $n_{\text {down }} \propto n_{\text {up }}{ }^{\sim 1}, T_{\text {down }} \propto n_{\text {up }}{ }^{-\sim 1}$, and also that the downstream density never exceeds the upstream density, $n_{\text {down }}<n_{u p}$, while in the 2D 
configuration usually we observe $n_{\text {down }}>>n_{u p}$. Figure 4 shows the evidence of a modest dependence, where the divertor density and temperature are plotted as a function of upstream density for LHD, W7-AS and TEXTOR-DED. The upstream densities in LHD, W7-AS and TEXTOR-DED are taken at the LCFS, where the poloidal variation of the density is usually small.

The phenomena have been interpreted as due to the loss of //-momentum as shown in Fig.2, which was first investigated in the island divertor configuration of W7-AS [96], using the 3D edge transport code EMC3-EIRENE [100,47]. In the W7-AS island divertor, due to the smallness of the island, the counter-streaming plasma flows along the island fans interact with each other and lose //-momentum, as discussed in the Section 2. The effect is elucidated in Fig. 5 by including the $\perp$-loss term in the momentum equation along flux tubes. The momentum loss factor, $f_{m}$, is defines as, $p_{\text {up }}=2 p_{\text {down }}\left(1+f_{m}\right)$, where $p_{\text {up }}$ and $p_{\text {down }}$ are the thermal pressure of ions and electrons at upstream and downstream, respectively [76]. The factor has a parameter dependence as $f_{m}=f_{m 0} / T_{d o w n}^{1 / 2}$ with $f_{m 0}$ representing the strength of the momentum loss. One sees that with an increasing momentum loss $\left(f_{m 0}\right)$ the upstream and downstream coupling becomes weak and then the density dependence changes from $n_{\text {down }} \propto n_{\text {up }}{ }^{3}, T_{\text {down }} \propto n_{\text {up }}{ }^{-2}$ to $n_{\text {down }} \propto n_{\text {up }}{ }^{\sim 1}, T_{\text {down }} \propto n_{\text {up }}{ }^{-\sim 1}$. An enhanced loss of //-momentum has also been identified in the stochastic layer of LHD [63]. In LHD, the interaction of counter-streaming flow is caused both by the radial compression of flux tubes near helical coils and the strong deformation of counter-directional flux tubes (i.e., flows) due to the strong magnetic shear $[63,101,102]$. Figure 6 shows an example of the counter streaming flow obtained by EMC3-EIRENE in the stochastic layer of LHD [63] together with the detection of such flow alternation with scanning Mach probe measurements [101]. In the figure, one sees that the counter-streaming flows, represented by yellow for positive and by blue for negative in toroidal direction, are compressed in a few $\mathrm{cm}$ range around the midplane ( $\mathrm{Z} \sim 0 \mathrm{~m}$ ). Numerical analysis for some configurations in HSX has also shown the absence of a high recycling regime due to the momentum loss in the edge island structure [103]. 
The effect of //-momentum loss can be formulated as the ratio between the momentum transport time parallel and perpendicular to the magnetic field,

$\frac{\tau_{m / /}}{\tau_{m \perp}}=\frac{D_{\perp} L_{/ /}}{V_{/ /} \lambda_{m}{ }^{2}}$,

where $D_{\perp}, L_{/ /}, V_{/ /}$are the perpendicular particle diffusivity, //-characteristic scale length for momentum transport, respectively. $\lambda_{m}$ is the $\perp$-distance between the counter-streaming flows, as defined in Section 2. The ratio corresponds to the momentum loss factor, $f_{m}$, discussed in refs.[104,63]. The larger the ratio is, the larger the $\perp$-loss of //-momentum is.

\subsection{Absence of high recycling regime due to enhanced $\perp$-energy transport or to enhanced convective energy flux}

In TEXTOR-DED with the $m / n=6 / 2$ mode of MP operation, the detailed analysis of the 3D edge transport simulation has shown that the replacement of //-energy flux $\left(q_{/ / e}\right)$ with $\perp$-transport $\left(q_{\perp e}\right)$, as discussed in Fig.1, is responsible for the modification of this density regime [105]. The enhanced $q_{\perp e}$ leads to the reduction of $/ /$-conduction energy $\left(q_{/ / e \text {, cond }}\right)$ and then reduces the parallel temperature gradient, which is required to carry the energy with the conduction. That is, $T_{\text {up }} / T_{\text {down }} \propto f_{\text {cond }}^{6 / 7}$, as elucidated in ref.[83], where $f_{\text {cond }}$ represents the fraction of the conduction component out of all of the parallel energy flux. Thus, this effect leads to a suppression of density increase at the divertor, such that $n_{\text {down }} \propto f_{\text {cond }}^{6 / 7}$, due to a pressure conservation along flux tubes. The contribution of $q_{\perp e}$ can be formulated as follows [104]: In the stochastic layer or in the island geometry, the radial energy transport for electrons can be written as,

$$
q_{r}=q_{\perp e}+q_{/ / e}=-n \chi_{\perp e} \frac{\partial T_{e}}{\partial r}-\kappa_{e 0} T_{e}^{2.5} \frac{\partial T_{e}}{\partial l} \vec{r} \cdot \vec{b}
$$

where $q_{r}, \chi_{\perp e}, \kappa_{0 e}$ and $l$ are the electron energy flux coming from the core, the perpendicular heat diffusivity and the coefficient for //-heat conductivity and the coordinate 
along the field line, respectively. $q_{r}$ can be delivered by two components, i.e. the first and second terms on the right hand side that represent the $\perp$-energy conduction and the radial projection of the //-energy conductive flux, respectively. $\vec{r}$ and $\vec{b}$ represent base vectors in the radial direction and tangential to the local magnetic field line as shown in Fig.1. Noting that $\frac{\partial}{\partial l}=\vec{b} \cdot \vec{r} \frac{\partial}{\partial r}$, the equation reads,

$$
q_{r}=q_{\perp e}+q_{/ / e}=-\frac{n \chi_{\perp}}{\vec{r} \cdot \vec{b}} \frac{\partial T_{e}}{\partial l}-\kappa_{e 0} T_{e}^{2.5} \frac{\partial T_{e}}{\partial l} \vec{r} \cdot \vec{b}
$$

One then obtains the ratio of perpendicular to parallel energy flux,

$$
\frac{q_{\perp e}}{q_{/ / e}}=\frac{n \chi_{\perp e}}{\left(B_{r} / B_{t}\right)^{2} \kappa_{0 e} T_{e}^{2.5}},
$$

here $\vec{r} \cdot \vec{b}$ is replaced with $B_{r} / B_{t}$. It is seen that under high density and low temperature condition the $\perp$-energy flux (the first term in eq.(3)) can provide a channel through which the //-energy flux (the second term) can be reduced.

In ref.[105], the replacement of //-energy flux $\left(q_{/ / e}\right)$ with the convection flux $\left(q_{/ / e \text {, conv }}\right)$ due to the substantial upstream ionization source, is also discussed as a cause for the density regime modification. The effect of convection energy flux was also pointed out in ref.[106]. This effect also leads to the reduction of //-conduction energy $\left(q_{/ / e \text {, cond }}\right)$ and reduces the parallel temperature gradient as discussed above. The effect of convection energy flux, $q_{/ / e \text { e, conv }} / q_{/ / \text {e, cond }}$ is strongly dependent on the detailed distribution of ionization distribution of plasma and thus the plasma parameters and the divertor geometry, which affect the neutral penetration into the edge plasma as discussed in details in $[11,107,108,93]$. In TEXTOR-DED, which has an "open” divertor configuration, the neutral penetration into the upstream region approaches $\sim 50 \%$ of the total recycling flux $[99,105]$. This upstream source gives rise to a substantial convection flux, $q_{/ / e \text {, conv }} / q_{/ / e \text {, cond }} \sim 1$ [105]. The estimation of $q_{/ / e \text { e, conv }} / q_{/ / \text {e, cond }}$ is, however, not straightforward and one needs detailed numerical analysis taking into account the 3D geometry of the edge plasma and of divertor and baffle structures. 


\subsection{Multi-machine comparison}

According to the above arguments, the larger the $q_{\perp e} / q_{/ / e}$ or $q_{/ / e, \text { conv }} / q_{/ / e \text {, cond }}$, the weaker the density dependence of the divertor plasma parameters, approaching $n_{\text {down }} \propto n_{\text {up }}^{\sim 1}, T_{\text {down }} \propto n_{\text {up }}^{-\sim 1}$.

In some cases of the 3D divertor configurations, however, the strong upstream and downstream coupling, i.e., the high recycling regime, is recovered, if either or both of $\tau_{m / /} / \tau_{m \perp}$ and $q_{\perp e} / q_{/ / e}$ are considered to be small enough to maintain the robust upstream and downstream coupling. This has been achieved in Tore Supra [109], TEXTOR-DED with an $\mathrm{m} / \mathrm{n}=3 / 1$ mode [110]. The numerical simulations with EMC3-EIRENE on $\mathrm{W} 7-\mathrm{X}$ and on HSX with a large island size have also shown a clear high recycling regime [62,103].

The calculation of $\tau_{m / /} / \tau_{m \perp}$ and $q_{\perp e} / q_{/ / e}$ for each device has been done as follows.

\subsubsection{Estimation of $\lambda_{m}$, the $\perp$-distance between the counter-streaming flows}

For the process of loss of parallel momentum, we consider a frictional interaction between counter-streaming flows, which are created in the edge region due to the magnetic field structure. It is found, from the numerical simulations in various devices, that the counter-streaming flow are formed not only in island divertors, but also in the stochastic layer of LHD [63], TEXTOR-DED [105], Tore Supra [111], DIII-D [112] and ITER [29]. It is also found that all these flow structures are regulated by the main poloidal mode number, $m$, of the perturbation field in the poloidal cross section. This formation of the counter-streaming flows is caused by the geometry of the flux tubes that are perturbed by the MP field, and is, therefore, mostly affected by the main mode number of the MP as it has the largest perturbation amplitude.

On the other hand, there always appears a fractal property in the magnetic field structure [37]. Those are created by an overlapping of island chains of higher order mode numbers induced by side band components of MP with usually significantly reduced amplitude. It is, 
however, an open issue to which small scale the fractal structure affects the plasma transport, while the present fluid model simulation can not correctly resolve any structure smaller than an ion Larmor radius. Also in the experiments, it is at present difficult to detect such a structure of plasma parameters in a scale small enough to assure the effects of fractal property. On the other hand, the flow alternation due to the main mode number is clearly observed in LHD with the Mach probe measurements [101], as shown in Fig. 6. Also in Tore Supra, a direct comparison of a Mach number between the simulations and the experiments has shown a reasonable agreement each other at least for the global structure [113].

While we don't know how much contributions are expected from the smaller structure (fractal property) of the higher mode to the main and the largest structure of the flow field, we may assume that the global momentum balance is still governed mainly by the largest flow field structure that is created by the main mode number as detected by both the simulations and the experiments. Based on these arguments, we define $\lambda_{m}$, the $\perp$-distance between the counter-streaming flows, as $\lambda_{m} \approx 2 \pi a / m$ for the present analysis, where $m$ is a poloidal mode number of the perturbation field. As for the effects of the fractal structure, we still have to wait for a refined modelling with a finer gird resolution including a kinetic effect, and for a measurement to resolve a plasma parameter distribution to assure an existence of such fractal structure in a reality.

An exception is introduced only for LHD, where the divertor leg flux tubes are stretched out of the edge stochastic region and deformed strongly due to the "local" magnetic shear $[12,63]$. The field lines are strongly sheared and directed largely in poloidal direction at the last step of the connection to the divertor plates. This deformation results in a much closer contact of counter-streaming flows than estimated with $\lambda_{m} \approx 2 \pi a / m$, as shown in Fig.6. This is a feature specific only to LHD, for which we have to modify $\lambda_{m}$ as $\lambda_{m} \approx 1 \mathrm{~cm}$ in order to correctly account for the momentum loss effect.

\subsubsection{Estimation of $L_{/ /}$, //-characteristic scale length for momentum transport}


Parallel flow profiles along flux tubes were investigated with the 3D numerical simulation in LHD. It has been found that the profile shows oscillation with a field period of $20 \sim 30 \mathrm{~m}$. In LHD, $2 \pi R q=24 \mathrm{~m}$. Therefore, the //-characteristic length of the flow field corresponds to roughly one poloidal turn of flux tube excursion. This correlation between the characteristic length of the oscillation and $2 \pi R q$ is interpreted as follows: Although the edge region becomes stochastic by an overlapping of magnetic island chains, still main trajectories of flux tubes follow the magnetic field direction defined by $B_{t}$ and $B_{\theta}$, since $B_{t}>>B_{\theta}>>B_{r}$. The flow field, therefore, can be affected significantly by a poloidal modulation of pressure and particle source distribution. In LHD, there is an in- and out-board asymmetry of the pressure distribution due to a toroidal effect and to a coil configuration. There is also an in- and out-board asymmetry in a particle source due to a divertor particle deposition pattern, which is usually intensive at in-board side. These poloidal modulation has $\mathrm{m}=1$ structure as the largest perturbation with additional higher order perturbations due to the remnant island structure. This results in such a flow profile reflecting the poloidal modulation of the pressure and particle source distribution. Similar correlation of the flow profile and $2 \pi R q$ is also found in DIII-D [114]. It is interpreted as due to the same reason as in LHD based on the pressure and particle distributions along poloidal direction in the single-null X-point divertor configuration. For the present analysis, therefore, we assume that $L_{/ /} \approx 2 \pi R q$. For the island divertor configurations, W7-AS/X and HSX, on the other hand, the connection lengths inside the edge island are used for $L_{/ /}$, since each island SOL is well separated each other and the flow field from the upstream (LCFS) to the divertor plates is considered not being perturbed significantly. Therefore, $L_{/ /}=L_{C} \sim 100 \mathrm{~m}[95,115]$ and 23 60 m [103], are used for W7-AS/X and HSX, respectively.

\subsubsection{Estimation of $\tau_{m / /} / \tau_{m \perp}$ and $q_{\perp e} / q_{/ / e}$}

For the calculations of $\tau_{m / /} / \tau_{m \perp}$ and $q_{\perp e} / q_{/ / e}$, the half values of the plasma parameters at the LCFS, i.e., $0.5 \times T_{L C F S}$ and $0.5 \times n_{L C F S}$, and a Mach number of 0.5 for 
the calculation of $V_{/ /}$, are assumed as representative values. Since the precise estimation of $\tau_{m / /} / \tau_{m \perp}$ and $q_{\perp e} / q_{/ / e}$ needs to be done by integration along the $3 \mathrm{D}$ structure of the SOL from the upstream to the downstream, neither the values at the LCFS nor at the divertor target are very appropriate for the calculation. Instead, here we assume that the intermediate value is as reasonable as possible for the estimation.

The model used for the present analysis is rather simplified, which has neglected some detailed features specific to each device. Nevertheless, we would like to note that the present analysis aims to obtain an overview of the transport characteristics in the 3D SOL geometry appearing in various devices, where a certain simplification of the model is inevitable in order to apply it for all the devices. The model has to be improved in future step by step, although the present analysis is the best that we can do at present using the available data from the different devices. While we are aware that there is a limitation of the present model for accurate evaluation of SOL characteristics, the simplification enables us to obtain an overview of the global trend of the SOL features of each device, which is our main purpose of the present study. Certainly, more sophisticated model together with a coordinated experimental program between different devices will provide much better analysis. Such works should remain for future tasks. The present analysis is, therefore, should be considered as very rough estimation and a kind of a test to see how the simple model can work out for describing the 3D effects, and used to see the qualitative tendency of the SOL characteristics of each device.

\subsubsection{Global trend of the divertor density regime}

Figure 7 summarizes the density regime of various devices plotted in the parameter space of $\tau_{m / /} / \tau_{m \perp}$ and $q_{\perp e} / q_{/ / e}$, where the results from numerical simulation are plotted with dashed lines. We have restricted the operation domain for the plot of each device to the upper half of the density range, taking into account the fact that the high recycling regime usually appears at high density range. The blue and red circles represent the cases with and without high recycling regime, respectively, while the black circles represent cases for which the existence of the high-recycling regime has not been confirmed. 
W7-AS covers rather wide range in $q_{\perp e} / q_{/ / e}$, from 0.1 to $\sim 10$. This means that the effect of perpendicular electron energy transport becomes significant only at the high density end of the operating domain in W7-AS. In other words, the 3D effect in terms of $q_{\perp} / q_{/ /}$ appears only at the high density end. On the other hand, W7-AS has rather high $\frac{\tau_{m / /}}{\tau_{m \perp}}$, and is located in the region, where all other devices belongs to the case without a high recycling regime. It has been also shown that there is no high recycling regime observed in W7-AS in both the experiments and in the simulations. The results indicate that in W7-AS the momentum loss due to the interaction of counter-streaming flows is a dominant process to affect the divertor density regime already at the lower density range, while at the higher density range the both momentum loss and the perpendicular energy flux affect the density regime.

Figure 7 shows a clear tendency of the transition from cases capable of obtaining the high recycling regime to cases with an absence of a high recycling regime as $\tau_{m / /} / \tau_{m \perp}$ and $q_{\perp e} / q_{/ / e}$ are increased. This suggests that the parameters $\tau_{m / /} / \tau_{m \perp}$ and $q_{\perp e} / q_{/ / e}$ can serve as an indication of the divertor density regime. From Fig. 7, the operation domain for the high recycling regime might be characterized as, $\left(\frac{\tau_{m / /}}{\tau_{m \perp}}\right)^{2}\left(\frac{q_{\perp e}}{q_{/ / e}}\right)<3.6 \times 10^{-5}$. Possible impacts on divertor functions due to the loss of the high recycling regime would be degradation of pumping efficiency and increase of physical sputtering due to the slow decrease of divertor temperature ( $T_{\text {down }} \geq 10$ during the attached phase). But these issues could be solved if the divertor/baffle geometry is properly designed to meet the engineering requirements for pumping, and if the detached phase is achieved, where the divertor temperature is reduced further. Another possible effect is a shift of detachment transition density to higher range due to the weak scaling of $T_{d o w n}$ with $n_{u p}$.

In the present analysis, we have used the divertor plasma density obtained from Langmuir probe measurements as an indication of whether the density dependence is in the 
high-recycling regime or not, instead of divertor particle flux, which might be a more robust quantity [87]. We have confirmed that the same feature is observed also in the particle flux measurements in the experiments [97,99]. It is also noted that the divertor plasma temperature is relatively high, $\gtrsim 10 \mathrm{eV}$ before the detachment transition for all the data referred to in the present analysis. Therefore, the estimation of the divertor density is considered to be reliable.

\subsection{Discussion on the case of $X$-point poloidal divertor}

3D numerical simulation has shown that in the case of an X-point poloidal divertor tokamak with an MP, for example DIII-D and ITER, the counter streaming flows also appear, as shown in Fig. 8. The figure plots the Mach number distribution of ITER with an MP [29]. It is noted that for the case of ITER, it has been shown that the strong magnetic shear deforms the flux tubes significantly and reduces $\lambda_{m}$ down to $\sim$ several $\mathrm{cm}$ at the high field side and the top of the plasma [29], as shown in Fig. 8. If we take the small value for $\lambda_{m}$, then $\tau_{m / /} / \tau_{m \perp}$ for the ITER case with an MP will increase and become closer to the border between the cases with and without high recycling regimes in Fig. 7. However, there is a fundamental difference between the X-point poloidal divertor with an MP and the other devices, W7-AS/X, Tore Supra, TEXTOR-DED, etc., as shown in Fig.1. In the former case, the dominant diverting field is still $B_{\theta}$, since $B_{\theta} / B_{t} \sim 0.1$ and $B_{r} / B_{t} \sim 10^{-3}$. In the latter case, the radial projection of //-flux due to $B_{r}$ directly competes with the $\perp$-transport. As long as one is considering //-flow, in the case of X-point poloidal divertor tokamaks with an MP, the flow direction might still be predominantly determined by $B_{\theta} / B_{t}$ with a small modulation due to $B_{r} / B_{t}$, and it might not cause significant effects on the //-momentum transport. Indeed, the density dependence of the divertor recycling flux calculated for the ITER with MP indicates nonlinear behaviour [116]. The computation of DIII-D with MP case also implies nonlinear density dependence of recycling flux [117]. Also the analysis of the divertor particle and heat flux distributions in ASDEX-Upgrade show no large difference in the global structure of the distributions in both computation and experiment between the cases with \& without MP, except for small modulations on the profiles [65]. The results indicate 
that the divertor recycling is not significantly affected by MP application. This is consistent with the indication of the ITER and DIII-D computations.

However, the flow field formation is a complex process depending on the ionization distribution as well as on drift as discussed in ref.[11]. In addition, there have been discussions on the energy and particle loss into the private flux region (PFR) in the 2D poloidal divertors, which affects the particle and energy deposition profiles on the divertor plates $[11,118,119,120,121]$. Recently the high-speed camera measurements have shown that propagating filaments eject plasma deeper into the PFR. [122]. However, fewer discussions are made on the possible effects on momentum transport. We need further detailed investigation of these issues both in experiments and numerical simulations.

\section{3D effects on SOL \& divertor impurity transport}

\subsection{Observations of core decontamination or impurity screening with edge stochastic layer or with island divertor}

There have been many observations of impurity screening or core decontamination in tokamaks with MP application [22,23,123,124,125,111,126], and in helical devices with a stochastic layer $[127,128,129,130,131,132,133,134]$ or with the island divertor configurations $[135,75]$. The evidence of the decontamination or screening effects are found for several impurity species, e.g. for carbon [22,123,124,125,111,126,127,132,135,75,133,134], nitrogen [22,23], oxygen [22,23,123], neon [131], argon [22,23] and for iron [128,130,131]. For neon, however, ref.[22,23] reports no screening effects. It has been also observed that the decontamination or the screening effect becomes better at higher density range. Fig.9 shows some of the experimental observations of carbon impurity behaviour with the edge stochastic layer as a function of density. In the figure one sees that carbon content in core plasma decreases with increasing density [111,126,127].

The systematic experiments in LHD varying the radial thickness of the stochastic layer have shown that the thickness of the stochastic layer has substantial effect on the impurity behaviour [132,136]. In Fig.10, shown is the ratio of CV+CVI to CIII+CIV as a function of density [132]. The interpretation of the ratio is as follows: There is a big gap in the ionization 
potential of carbon between $\mathrm{CV}\left(\mathrm{C}^{4+}\right)$ and $\mathrm{CIV}\left(\mathrm{C}^{3+}\right)$, i.e. $\mathrm{CIII}\left(\mathrm{C}^{2+}\right): 48 \mathrm{eV}, \mathrm{CIV}\left(\mathrm{C}^{3+}\right): 65 \mathrm{eV}$, $\mathrm{CV}\left(\mathrm{C}^{4+}\right): 392 \mathrm{eV}$, CVI $\left(\mathrm{C}^{5+}\right): 490 \mathrm{eV}$, respectively. Thus, the emission from the higher charge states $(\mathrm{CV}+\mathrm{CVI})$ is a proxy of carbon content in the core, and that from the lower charge states (CIII+CIV) is a proxy of carbon at the very edge region or the source. Therefore, it is considered that the reduction of the ratio indicates an impurity screening effect, and it is seen that the thicker stochastic layer has a clear screening already in a lower operating density range compared to the thin stochastic layer. A similar tendency has also been observed in TEXTOR-DED. The screening effect is clearly observed in the case of $m / n=3 / 1$ mode, which provides stochastic layer with thickness of $\sim 0.20 \mathrm{~m}$ (table 1 ). On the other hand, in the case of 12/4 mode, which gives only $\sim 0.05$ m thickness of stochastic layer (table 1 ), there is no clear evidence of screening found [126]. The results imply that the thicker stochastic layer can provide better screening than the thinner one.

\subsection{Parameterization of impurity screening by the 3D effects}

It is not clear yet at all whether the above phenomena are caused by the edge impurity screening or attributed to core/pedestal transport, or some other effects such as a change of source characteristic, etc. In this analysis, however, we focus on the 3D geometrical effects on the edge impurity transport in the SOL/divertor region. A simple picture of impurity screening in the 3D divertor configuration may be drawn as follows: Based on the //-momentum balance equation for the impurity, the resultant profile of the impurity density is considered to be determined by a competition between the friction force and the ion thermal force acting on the impurity [83]. That is,

$$
m_{z} \frac{\partial V_{z / /}}{\partial t} \approx m_{z} \frac{V_{/ /}-V_{z / /}}{\tau_{s}}+C_{i} \nabla_{/ /} T_{i}+\ldots,
$$

where $m_{z}, V_{z / /}, V_{/ /}, \tau_{s}$ are the mass of the impurity, the //-velocity of the impurity, the //-velocity of background plasma, and the collision time (slowing down time of the impurity) between the impurity and the plasma, respectively. $C_{i}$ is a coefficient for ion thermal force, where $C_{i} \rightarrow 2.6 Z^{2}$ for $m_{z} /\left(m_{z}+m_{i}\right) \rightarrow 1$. The friction force drags the impurity towards the plasma flow direction (for example, towards the divertor region, assuming that the plasma 
flow is directed towards the divertor plates). On the other hand, the ion thermal force drives the impurity upstream because the //-temperature gradient points upstream and toward higher temperatures.

With $B_{r}$ from the MP, the outward (radial) plasma flow can be enhanced due to the radial projection of $V_{/ /}$. This also leads to an enhancement of recycling due to a reduced particle confinement time, and thus results in a colder and denser edge region, which reduces the collision time between the plasma and the impurity, $\tau_{s}$ [83]. Since the friction term has the dependence of $\sim \frac{V_{/ /}-V_{z / /}}{\tau_{s}}$, the above effects lead to an enhancement of the friction force. It is also noted that effective screening is often observed in the higher density range, i.e., at higher collisionality $[111,127,128,129,126]$, as shown in Fig. 9. This is also consistent with the picture of the friction force that is inversely proportional to the collision time, $\tau_{s}$. The effects of the friction force have been pointed out so far in many publications $[137,138,139,140,141,142]$. Here we compare an enhancement of outward (radial) particle flux due to the radial projection of the //-flow, $\Gamma_{s t}^{p}=n V_{/ /}\left(B_{r} / B_{t}\right)$, with a radial projection of $\perp$-particle diffusion, $\Gamma_{r \perp}^{p} \approx-D_{\perp} \frac{\partial n}{\partial r}$, to measure the effect of friction force. That is,

$$
\frac{\Gamma_{s t}^{p}}{\Gamma_{r \perp}^{p}}=\frac{n V_{/ /}\left(B_{r} / B_{t}\right)}{-D_{\perp} \frac{\partial n}{\partial r}} \approx \frac{n V_{/ /}\left(B_{r} / B_{t}\right)}{D_{\perp} \frac{n}{\delta r}}=\frac{V_{/ /}\left(B_{r} / B_{t}\right) \delta r}{D_{\perp}}=\frac{\left(B_{r} / B_{t}\right)^{2} V_{/ /} L_{/ /}}{D_{\perp}}
$$

where $\delta r$ is a $\perp$-scale length of particle transport, for which we have assumed a relation, $\delta r=L_{/ /}\left(B_{r} / B_{t}\right)$, and $D_{\perp}$ is the $\perp$-particle diffusivity, which ranges from 0.4 to $1.0 \mathrm{~m}^{2} / \mathrm{s}$, depending upon the device (table 1). It is noted that, the formula also can be a measure of

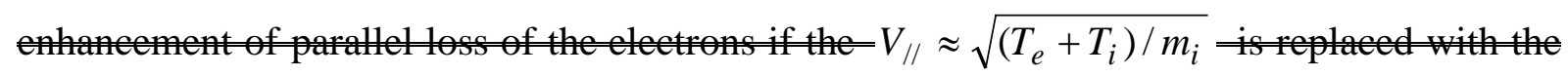
electron thermal velocity $v_{\text {th, } e} \sim \sqrt{T_{e} / m_{e}}$ If assume that $T_{e} \sim T_{i}$, then $v_{\text {th }, e} \propto V_{/ /}$\%

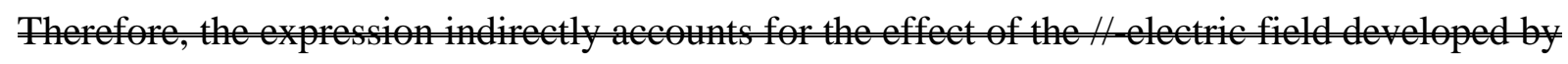
the fast escaping electrons due to the open field lines in the stochastic field or in the island diveror. The $/$ electic field also pushes the impunity tow the diveror plates. 
One should also note that a highly collisional plasma also develops a //-temperature gradient, and thus an ion thermal force. Here the same argument for electrons in eq.(5) in Section 3.2 can apply for ions. Therefore, in the island structure that appears in the 3D divertor configurations, the //-temperature gradient is reduced by increasing $\perp$-conductive energy flux under high density and low temperature conditions. It has been shown that this effect becomes remarkable when $n \chi_{\perp}>\kappa_{i 0} T_{i}^{2.5}\left(B_{r} / B_{t}\right)^{2}$ [104]. It thus leads to a suppression of the ion thermal force, which is proportional to the //-temperature gradient. This effect has been pointed out in the numerical simulation with EMC3-EIRENE on W7-AS [104] and also later confirmed in LHD [127]. The effect can be formulated as [76,104],

$$
\frac{q_{\perp i}}{q_{/ / i}}=\frac{n \chi_{\perp i}}{\left(B_{r} / B_{t}\right)^{2} \kappa_{0 i} T_{i}^{2.5}}
$$

similar to eq.(5) but now for ions. In the case of a 2D axi-symmetric X-point divertor, where $B_{\theta} / B_{t} \sim 0.1$ is used instead of $B_{r} / B_{t}$ as discussed at eq.(1) in Section $2, \frac{q_{\perp i}}{q_{/ / i}}<1$. Therefore, the suppression of ion thermal force is not foreseen. In the 3D divertor, on the other hand, due to a very small $B_{r} / B_{t}=10^{-4} \sim 10^{-3}$, the condition $\frac{q_{\perp i}}{q_{/ / i}} \sim 1$ is fulfilled.

The modelling analysis that takes into account these effects has been conducted in comparison with the carbon emission measurements in LHD [127], where qualitatively good agreement has been found for the CIV profile and density dependence of CIII, CIV, and CV.

As shown in Fig.10, the thickness of the stochastic layer of the edge island, $\lambda_{s t-S O L}$, can be a parameter to characterize the screening effect. This is considered to be due to an increase of the radial extension of the friction force dominant region, which is developed in the stochastic region as discussed above. We parameterize this effect by normalizing with impurity penetration length, $\frac{\lambda_{\text {st-SOL }}}{\lambda_{\text {imp }}}$, where $\left.\lambda_{\text {imp }}=V_{i m p}^{0} /(<\sigma v\rangle_{\text {ion }} n\right) \cdot V_{\text {imp }}^{0}$ and $\langle\sigma v\rangle_{\text {ion }}$ are the velocity of the neutral impurity and a rate coefficient of neutral impurity ionization, respectively. 


\subsection{Multi-machine comparison}

Figure 11 (a) summarizes the observations of impurity screening in experiments or in numerical simulations, in terms of $\frac{\Gamma_{s t}^{p}}{\Gamma_{r \perp}^{p}}$ and $\frac{\lambda_{\text {st-SOL }}}{\lambda_{\text {imp }}}$. For $\lambda_{\text {imp }}=V_{i m p}^{0} /\left(\langle\sigma v\rangle_{\text {ion }} n\right)$, we used $0.05 \mathrm{eV}$ for the neutral impurity injection energy and $0.25 T_{L C F S}, 0.25 n_{L C F S}$ as representative plasma parameters for calculations of the index. The selection of the factor 0.25 , instead of 0.5 as done in Section 3, is to take into account the fact that neutral impurities come from outside of the plasma and interact with plasma in the more peripheral regions. Due to the same arguments in Section 3, however, this should be considered as a very rough estimate to obtain an overview of the SOL characteristics between different machines. More precise calculations using the 3D plasma structure should be carried out in the future. In the present analysis, carbon is assumed as the impurity. In Fig. 11 (a), we have plotted the results of the upper half of the operation range of density, taking into account the fact that the screening effects are pronounced always at high density operation [124,111,104,76,127,128,126], as discussed above. In Fig.11 (a), there is a clear tendency that if we increase $\frac{\Gamma_{s t}^{p}}{\Gamma_{r \perp}^{p}}$ or $\frac{\lambda_{s t-S O L}}{\lambda_{\text {imp }}}$, the screening effects emerge. This tendency has been overlooked in the preceding analysis [143], where the density effect on the screening has not been taken into account.

The loss of //-momentum, as discussed in Section 3, affects the friction force effect, as it is due to a flush out of impurity by plasma flow. The momentum loss effect enters to the present analysis as follows: The equation (7) can be translated as,

$$
\frac{\Gamma_{s t}^{p}}{\Gamma_{r \perp}^{p}}=\frac{\left(B_{r} / B_{t}\right)^{2} V_{/ /} L_{/ /}}{D_{\perp}}=\frac{V_{/ /} L_{/ /}^{2}}{D_{\perp} L_{/ /}}\left(B_{r} / B_{t}\right)^{2}=\frac{V_{/ /} \lambda_{m}^{2}}{D_{\perp} L_{/ /}} \frac{L_{/ /}^{2}}{\lambda_{m}^{2}}\left(B_{r} / B_{t}\right)^{2}=\frac{1}{\tau_{m / /} / \tau_{m \perp}}\left(\frac{\delta r}{\lambda_{m}}\right)^{2},
$$

where $\delta r=L_{/ /}\left(B_{r} / B_{t}\right)$ is a $\perp$-scale length of particle transport as defined above. $\delta r$ can be also viewed as a radial deflection effect of the //-transport due to the perturbation field. Since the momentum loss effect, $\tau_{m / /} / \tau_{m \perp}$, enters to the denominator of the formula, the enhancement of the //-momentum loss reduces the friction force effect, $\Gamma_{s t}^{p} / \Gamma_{\perp r}^{p}$. On the other 
hand, the enhancement of radial component of transport, $\delta r$, enters to the numerator of the formula. Thus $\Gamma_{s t}^{p} / \Gamma_{\perp r}^{p}$ represents a competition between the reduction of friction force due to the //-momentum loss and the enhancement of radial transport caused by the magnetic field deflection due to a perturbation field.

In order to see the effects of the two terms, $\tau_{m / /} / \tau_{m \perp}$ and $\left(\delta r / \lambda_{m}\right)^{2}$, separately, we have plotted the operating range of each device in a $\tau_{m / /} / \tau_{m \perp}-\frac{\lambda_{s t-S O L}}{\lambda_{i m p}}$ and a $\left(\delta r / \lambda_{m}\right)^{2}-\frac{\lambda_{\text {st }-S O L}}{\lambda_{\text {imp }}}$ spaces, as shown in Fig.A1 (Appendix). In Fig.A1 (a), it is not clear if a smaller momentum loss $\left(\tau_{m / /} / \tau_{m \perp}\right)$ is better for screening, since at the same range of $\tau_{m / /} / \tau_{m \perp}$, i.e. $\tau_{m / /} / \tau_{m \perp}=10^{-3} \sim 10^{-2}$, the cases both with and without impurity screening are populated. On the other hand, even at the higher $\tau_{m / /} / \tau_{m \perp}$ domain, the impurity screening cases of W7-AS and LHD are found. Only a trend found from the figure is that the devices with the screening tend to be located at the higher $\frac{\lambda_{s t-S O L}}{\lambda_{\text {imp }}}$ region. Also it is noted that LHD is located at the highest $\frac{\lambda_{s t-S O L}}{\lambda_{\text {imp }}}$ domain except for ITER.

From Fig.A1 (b), on the other hand, the larger $\left(\delta r / \lambda_{m}\right)^{2}$ and the larger $\frac{\lambda_{s t-S O L}}{\lambda_{\text {imp }}}$ result in the impurity screening cases, which is consistent with a picture of impurity screening by a friction force with the larger radial deflection of //-flow, and by the stopping effect of neutral impurities by the thicker SOL. It is also noted that LHD and W7-AS are located at rather large $\left(\delta r / \lambda_{m}\right)^{2}$ region. From the fact that W7-AS and LHD have either a larger $\left(\delta r / \lambda_{m}\right)^{2}$ or a larger $\frac{\lambda_{\text {st-SOL }}}{\lambda_{\text {imp }}}$, we could hypothesize that these two effects compensate the large momentum loss, resulting in the screening effects. 
As shown in Fig.11 (a), indeed W7-AS has larger $\frac{\Gamma_{s t}^{p}}{\Gamma_{r \perp}^{p}}$, which is consistent with the picture of impurity screening by the enhanced outward flow, as a result of competition between $\tau_{m / /} / \tau_{m \perp}$ and $\left(\delta r / \lambda_{m}\right)^{2}$. For LHD, on the other hand, $\frac{\Gamma_{s t}^{p}}{\Gamma_{r \perp}^{p}}$ turned out to be small. It could be thus interpreted that the large momentum loss is compensated by the thicker stochastic layer width to provide the screening by effectively stopping the impurity neutral penetration. It should be, however, noted that the above argument is at present a hypothesis, and we need more data points to clarify it.

The operation domain for effective impurity screening might be expressed as, $\left(\frac{\lambda_{s t-S O L}}{\lambda_{\text {imp }}}\right)\left(\frac{\Gamma_{s t}^{p}}{\Gamma_{\perp}^{p}}\right)^{1 / 4}>18$. A rather weak dependence on $\frac{\Gamma_{s t}^{p}}{\Gamma_{r \perp}^{p}}$ is found.

In TJ-II, a core decontamination has been observed by changing the edge magnetic field configuration from the standard one to a configuration similar to an island divertor $[75,144]$. The operation range of TJ-II is located at $\frac{\lambda_{s t-S O L}}{\lambda_{\text {imp }}} \sim 0.3$ and $\frac{\Gamma_{s t}^{p}}{\Gamma_{r \perp}^{p}} \sim 2.3$, which is outside of the plot range of Fig. 11 (a) and indeed outside of the domain of the impurity screening characterized with the expression above, showing inconsistency with other devices. Due to the small value of $\frac{\lambda_{\text {st-SOL }}}{\lambda_{\text {imp }}}$, less than unity, a substantial fraction of impurity might penetrate beyond the SOL region. In such a case the argument of the $3 \mathrm{D}$ effect on the SOL impurity transport discussed in the present analysis might not apply in TJ-II. The resultant impurity behaviour might depend substantially on the source characteristics or/and on the core transport due to the change of the magnetic field configuration, as pointed out also in ref. [144]. However, the systematic experiments in TJ-II by changing the radial location of impurity source indicate a clear effect of the edge island on the core impurity content $[75,144]$. The change of the core transport due to the configuration change is precluded in the ref. [75], too. On the other hand, the strong, perpendicular ripple-induced transport is pointed out in TJ-II, while no numerical calculations with an estimate of the radial electric field at the edge 
are presently available for this effect $[75,144]$. If this is the case, however, the inconsistency between TJ-II and other devices in terms of the present parameterization is not surprising, since such ripple effect is not taken into account in the present analysis. Based on the argument, here we accept the results of TJ-II as a caveat on the present analysis, rather than omitting them. The present considerably simplified analysis certainly can not capture all possible processes of impurity screening effects. In order to clarify the issue, we need more data accumulation from various devices.

A similar plot but with a horizontal axis replaced by $\frac{q_{\perp i}}{q_{/ / i}}$, which accounts for the thermal force suppression effect, is shown in Fig.11 (b), where no clear parameter dependence is observed with respect to impurity screening. It is noted that an increase in $\chi_{\perp}\left(=3 D_{\perp}\right)$ leads to an increase of $q_{\perp i} / q_{/ / i}$, i.e. a suppression of thermal force, but at the same time it results in an increase of momentum loss, i.e. a reduction of friction force effect. It is also noted that the term $\left(B_{r} / B_{t}\right)^{2}$ gives opposing effects between the friction force, $\Gamma_{s t}^{p} / \Gamma_{\perp r}^{p}$, and the thermal force, $q_{\perp i} / q_{/ / i}$, since the term, $\left(B_{r} / B_{t}\right)^{2}$, enters to the numerator and denominator of the formulae, respectively. This means that the screening effects are not monotonic function of $\chi_{\perp}$ and $\left(B_{r} / B_{t}\right)^{2}$, but there is optimum values to maximize the effect. We could not, however, identify such optimum operating range in the present analysis. This is partly because the whole system is controlled by the several parameters as discussed above, which are interacting each other, and also because probably there are still hidden parameters that are not taken into account at the moment, such as impurity source locations, drift, electric field etc. It is also important to confirm whether or not this thermal force suppression effect exists in the experiments, since the effect is at present predicted only in the numerical simulations. These works are still left for future tasks.

It should be mentioned that, as we surveyed $Z_{\text {eff }}$ data from various devices both of $2 \mathrm{D}$ axi-symmetric divertors $[145,146,147,148,149,150,151,152]$ and 3D divertor configurations $[124,111,135,153], Z_{\text {eff }}$ ranges between 1 to 4 , and there is no clear difference found between 
the $2 \mathrm{D}$ and $3 \mathrm{D}$ configurations, at the moment. It is also noted that the data shows clear differences depending on the divertor/wall materials, i.e. differences due to the changes from carbon to tungsten [149] or to an ITER like wall [148]. This means that the impurity species and the source characteristics play important roles in the impurity transport. Thus we still need further intensive analysis for clarifying the effects of the 3D divertor configuration. The following issues are still left for future works: the definition of screening efficiency, e.g. the ratio of impurity influx at the LCFS to the impurity source at the PFC, the plasma parameters (such as density or collisionality) dependence of the efficiency, the effects of impurity injection energy, recycling location, drift, electric field, and turbulent transport.

\section{3D effects on edge radiation and detachment stability}

\subsection{Tore Supra}

Because of the fast //-transport with respect to $\perp$-transport, change of magnetic geometry can modify the edge radiation structure. This has been demonstrated in the Tore Supra ergodic divertor with an MP applied to lower hybrid current drive (LHCD) discharges [154]. It has been found that with relatively low density, the Greenwald density fraction of 12 to 22\%, a MARFE is obtained during MP application with LHCD. In the discharge, the radiated power is almost doubled compared to the phase without an MP. The MARFE is positionally stable at the high field side with radiation more than $90 \%$ of the total input power [154]. Ha signals indicate plasma detachment from the high field side wall. It was found that the effects associated with the MARFE were controlled by adjusting the LHCD input power, MP amplitude, and the edge safety factor. The results indicate the importance of the edge magnetic field geometry on the edge radiation stability.

\subsection{W7-AS}

In W7-AS, it has been found that with proper choice of the edge island geometry, i.e., large island and with short connection length, an operational domain with steady state partial detachment is realized $[155,156]$. In such cases, the 3D numerical simulation with EMC3-EIRENE shows that the radiation region moves to the inboard side and is peaked around island X-points, as shown in Fig. 12 (a), where the carbon radiation distribution 
obtained by the 3D numerical transport simulation is plotted for the stable detachment case. On the other hand, in the case with a small island and a long connection length, the intense radiation remains in front of the divertor plate, as shown in Fig.12 (b). In this case the plasma stored energy significantly decreases after detachment transition and the radiated power strongly oscillates. Finally the discharge terminates due to a deep inward shift of the radiation front. We call this case unstable detachment in comparison to the steady state partial detachment mentioned above, which is referred to as stable detachment. The operation domain for stable detachment is characterized by the distance between the divertor plate and the LCFS, $\Delta x_{\text {LCFS-div }}$ (Fig.14), and $L_{C}$ [156], i.e. the lower $L_{C}$ and larger $\Delta x_{L C F S-d i v}$ are preferred for the stable detachment. The physical interpretation of the different behaviour between the stable and unstable detachment is as follows: In the case of unstable detachment, the divertor radiation lowers the temperature in the recycling zone and the island becomes transparent to the neutral penetration into the core region. It is considered that the sudden increase of neutral penetration into the core at the detachment transition drives an instability resulting in collapse. On the other hand, in the case of stable detachment with the larger island accompanied by the inboard side radiation instead of the divertor radiation, the island temperature is still high enough to have a screening effect against the neutral penetration and thus stabilizes detachment [156]. This is probably a similar argumentation to that often made in axi-symmetric tokamak devices, too $[157,158]$.

\subsection{LHD}

In LHD, it has been found that the application of an MP with $m / n=1 / 1$, which creates a remnant island structure in the stochastic region, has a stabilizing effect on the detachment [159]. Here the definition of the stable detachment is similar to that of W7-AS. The stable detachment means that the detached phase can be sustained with gas puff feedback control for more than several seconds until the end of the NBI heating with almost constant radiated power. Without an MP, the sudden increase of the radiation can not be stopped even if the gas puff is turned off, and the discharge ceases. We call this case unstable detachment. The divertor probe array measurements show that the particle flux profile has an $n=1$ mode structure in the toroidal direction during detachment [160]. The 3D numerical simulation with 
EMC3-EIRENE shows that with MP application intense radiation is formed along the trajectory of the X-point of the $m / n=1 / 1$ island, while without the MP the radiation is localized at the inboard side throughout the torus [159]. Figure 13 shows the resulting distribution of the carbon radiation obtained by the numerical simulation together with the radiation measurements in experiments. In the figure, one clearly sees the change of radiation pattern from the peaked inboard side radiation without MP (Fig.13 (b)) to the X-point radiation with MP application (Fig.13 (a)). As shown in Fig.13, the radiation distribution measurements with both AXUVD as well as imaging bolometer show the signature of the intense X-point radiation, confirming, at least qualitatively, agreement with the code prediction for the modification of the 3D radiation structure $[160,161,162]$. The operation domain for the stable detachment with an MP is found to be characterized by the distance between the island X-point and the LCFS, $\Delta x_{L C F S-i s l a n d}$ (Fig.14), and the MP amplitude, $B_{r} / B_{t}$ [159]. In order to realize stable detachment one needs both the larger $\Delta x_{L C F S-i s l a n d}$ and $B_{r} / B_{t}$, which are similar parameters to those mentioned in the W7-AS case.

\subsection{Comparison between W7-AS and LHD}

Figure 14 shows the operation domain for the stable detachment in LHD and W7-AS in the parameter space of $\Delta x_{L C F S-i s l a n d}\left(\Delta x_{L C F S-d i v}\right)$ and $B_{r} / B_{t}$. The figure shows that in order to obtain the stable detachment the MP field must be strong enough to modify the edge magnetic field structure and thus transport there, and the geometric SOL width or the separation between the radiation region and the confinement region must be sufficiently large. There exists a similar tendency between W7-AS and LHD with respect to $\Delta x_{L C F S-i s l a n d}$ in LHD and $\Delta x_{L C F S-d i v}$ in W7-AS. This indicates the importance of the decoupling between the core and the radiation regions or the divertor recycling regions to avoid instability caused by the sudden neutral penetration at the detachment transition. The stability analysis with a perturbation method also shows that flattening of the temperature profile at the island plays a key role to prevent inward penetration of radiation [163]. It is also found that the larger the $B_{r} / B_{t}$ is, the lower the detachment onset density [160], due to the enhanced radiation 
caused by the larger volume of radiation region in the edge island. This is a similar trend observed in the MARFE onset in Tore Supra [154] discussed above.

\subsection{TEXTOR-DED}

In TEXTOR-DED, it has been observed that application of a rotating MP to an NBI heated plasma shifts the MARFE onset density to a higher range compared to the case without an MP [164]. The experimental results show that the threshold of the MARFE onset strongly depends on both the level and the poloidal distribution of recycling at the high field side, where the MP coils are located. It is considered that both the smoothing of recycling and the enhanced poloidal flow of plasma particles with moving field lines of a rotating MP, as compared to the static MP, can increase the onset density of a MARFE by avoiding localized cooling of the plasma in front of the MP coils [164].

The experimental observations shown above, Tore Supra, W7-AS, LHD and TEXTOR-DED, clearly indicate a possibility to control the edge radiation structure and the detachment stability in $3 \mathrm{D}$ divertor configurations. The key controlling parameters are the distance between the edge radiation region, recycling region and the confinement region, $\Delta x_{L C F S-i s l a n d, d i v}$, the MP amplitude, $B_{r} / B_{t}$, and the rotating frequency of the MP, $f_{R M P}$.

\section{3D effects on edge electric field and turbulence}

The edge electric field formation is believed to be of crucial importance to control edge plasma turbulence by means of ExB sheared flows. Since the $\perp$-transport is considered to be governed by the turbulence, this then affects the SOL/divertor transport significantly. In addition, the electric field influences not only plasma but also impurity transport through drifts.

The change of the edge magnetic geometry by the application of an MP can affect turbulence via several effects caused by magnetic field braiding: the radial component of the field lines, $B_{r}$, can induce a radial electron current, induce radial transport such as $\Gamma_{s t}^{p}$ (eq.(7)). It can also modify the parallel wavelength of the modes, $k_{/ /}\left(\nabla_{/ /}=\left(\vec{B}_{0}+\vec{B}_{r}\right) \bullet \vec{\nabla}\right)$, 
can increase sheath dissipation through the open field lines, etc. Measurements and analysis of the radial electric field and fluctuations with MP application in tokamaks, helical devices and RFPs have been conducted in several devices, TEXTOR-DED [165,166,167], Tore Supra [50,168,169], TEXT [170,171], CSTN-III [172], MAST [173,174], ASDEX-Upgrade [175,176], DIII-D [177], LHD [178], and RFX-mod [179]. It is noted that the divertor density regime, impurity transport and detachment stability discussed above are affected through the //-momentum loss, $D_{\perp}, \chi_{\perp}$ via the turbulent transport, and through the impurity drift caused by the electric field.

In TEXTOR-DED, it has been observed that the MP application leads to the suppression of blob transport [180]. This is thought to be due to the suppression of large scale turbulence structure with MP application by changing the mode structure from $k_{/ /} \approx 0$ (without an MP) to finite $k_{/ /}$(with an MP), as observed in experiments [168,165], which then reduces the blob size. This is also confirmed in numerical simulations with ATTEMPT [181]. Moreover, the enhanced sheath dissipation, caused by the increased volume of open field lines with an MP, is responsible for the reduction of the blob radial velocity as observed in the numerical analysis in ref.[182].

In the experiments of TEXTOR-DED [183] and MAST [174], the reduction of the long range correlation of potential fluctuations during MP application has been observed, which suggests the reduction of zonal flows. Possible mechanisms for this effect might be the suppression of large turbulent structures with MP due to the nonzero $k_{/ /}$as mentioned above, and also the decrease in the Reynolds stress which is a drive for the zonal flows. However, observation in ASDEX-Upgrade showed that with an MP there is little change in the relative fluctuation level of the ion saturation current, $\delta I_{\text {sat }} / I_{\text {sat }}$, in the SOL [175]. The details of the mechanism are under investigation in refs.[184,185,186] with sophisticated models.

It is also noted that clear changes of the plasma potential profile as well as the edge electric field have been observed [165,166,167,173,171,175], where the radial electric field tends to change from negative (inward) to positive (outward). This is interpreted as due to the effect of open field lines produced by an MP, where the fast escaping electrons compared to the ions must develop a positive field to restore the ambipolarity. In DIII-D [177], the radial 
profiles of plasma potential, electron temperature, poloidal electric field, DC ExB flux and turbulent radial particle transport inside the LCFS are all modified by a MP when the C-coil current $\left(\mathrm{I}_{\mathrm{c}}\right)$ increases from 1 to $3 \mathrm{kA}$. On top of these, very recent experiments in ASDEX-Upgrade [176] have reported a variety of plasma responses to the MP application, i.e. the changes of electric field and of turbulent transport differ depending on the plasma parameters, MP modes and amplitude etc. In TEXTOR-DED, it is also observed that the particle confinement time changes with the MP application, which is correlated with the change of electric field [166,167].

These results suggest that the analysis of electric field formation and turbulent transport under MP application, including plasma response against MP penetration, is an urgent issue to be addressed for identification of the impacts of MP on the divertor functions and plasma-wall interaction.

\section{Discussions and Summary}

The effects of the 3D edge magnetic field geometry on the SOL and divertor transport have been discussed with regards to the divertor density regime, impurity screening, detachment control, and the edge electric field/turbulent transport. In the present assessment, the 3D effects are emergent when the $\perp$-transport starts to compete with //-transport in the open field lines of the stochastic layer or in the magnetic island structure, and thus delivering plasma quantities (particle, energy, momentum) from the upstream region (around the LCFS) to the divertor, i.e., $\Gamma_{/ /} \Theta \sim \Gamma_{\perp r}$ or $\Gamma_{\perp \theta}$, where $\Theta \equiv B_{r} / B_{t}$ or $B_{\theta} / B_{t}$. This condition is met in the stochastic layer or in the edge islands in the island divertor configurations, with typically $B_{r} / B_{t}=10^{-4} \sim 10^{-3}$. On the other hand, the condition is not met in the $2 \mathrm{D}$ axi-symmetric configuration with an X-point divertor because $B_{\theta} / B_{t} \sim 0.1$ and thus $\Gamma_{/ /}\left(\frac{B_{\theta}}{B_{t}}\right)>>\Gamma_{\perp \theta}$. For the vector field such as momentum transport, the direction of the field line connection to the divertor plates, i.e., plasma flow direction, becomes important. The spatial separation of opposite vector quantities, $\lambda_{m}$, the distance between the counter-streaming flows, is a key parameter to determine the importance of $\perp$ loss of //-momentum through viscosity between counter flows. 
Based on this picture, the controlling parameters of the 3D effects have been derived for the individual divertor functions. Table 2 summarizes the parameters together with the experimental observations, devices, physical interpretations and possible impacts on the divertor functions.

The two parameters are suggested for characterizing the divertor density regime. One is the ratio of momentum transport time in // to $\perp$ direction, $\frac{\tau_{m} / /}{\tau_{m \perp}}$, which represents the loss of //-momentum through viscosity between counter-flows. Another one is the ratio of energy flux in $\perp$ to // direction, $\frac{q_{\perp e}}{q_{/ / e}}$, which represents the replacement of //-energy flux with $\perp$-flux. Surveying the results from various devices, the two parameters appear to be a good measure for the presence or absence of the high recycling regime. It is noted that the interpretation applies to the attached phase only, while in a detached phase more complex physics come into play, such as atomic or molecular processes, ionization front shift to the upstream region etc. $[187,188]$. The absence of a high recycling regime, which can occur in the domain indicated in Fig.7 might have an impact on the pumping efficiency due to the low divertor (neutral) density as well as on the physical sputtering due to the slow decrease of the divertor temperature as a function of $n_{u p}$. These effects have to be discussed further taking into account the divertor and baffle structure, which affect the neutral distribution. Also the available pumping speed, fuelling efficiency and operating density range are all influenced by 3D plasma distributions. The slow decrease of the divertor temperature against the upstream density can shift the detachment onset to higher densities. The higher $n_{u p}$ is preferred for impurity screening by suppressing the ion thermal force as discussed in Section 4. The value of $n_{u p}$ also can affect core plasma confinement. The operation domain for the high recycling regime is found to be characterized as $\left(\frac{\tau_{m / /}}{\tau_{m \perp}}\right)^{2}\left(\frac{q_{\perp e}}{q_{/ / e}}\right)<3.6 \times 10^{-5}$.

With respect to the impurity screening or core decontamination with the 3D divertor configuration (stochastic edge or island divertor), it is found that the parameterization with the 
edge transport effects such as the friction force, $\frac{\Gamma_{s t}^{p}}{\Gamma_{r \perp}^{p}}$ and thickness of the stochastic layer or island divertor SOL, $\frac{\lambda_{\text {st-SOL }}}{\lambda_{\text {imp }}}$, can be a measure for the screening effect. This has been missed in the preceding analysis [143], where the density effect on the screening has not been taken into account. The operation domain for the impurity screening is found to be characterized as $\left(\frac{\lambda_{s t-S O L}}{\lambda_{\text {imp }}}\right)\left(\frac{\Gamma_{s t}^{p}}{\Gamma_{\perp}^{p}}\right)^{1 / 4}>18$. On the other hand, it is found that the parameterization with the ion thermal force suppression, $\frac{q_{\perp i}}{q_{/ / i}}$, does not work very well, although these effects have been discussed much in the literature. It should be also kept in mind that the results of TJ-II show inconsistency with other devices, posing a caveat on the present simplified model, which certainly can not capture all possible effects on impurity transport. Yet there are still several issues to be assessed for the impurity transport, i.e. dependence on impurity species, source location, injection energy, definition of screening (the ratio of impurity influx at the LCFS to the impurity source at the PFC), operation range for screening (density or collisionality dependence), effects of ripple, drift, electric field, turbulence and core transport. All these issues are still left to future works.

Figure 15 summarizes the 3D effects in terms of divertor density regime and the impurity screening based on the analysis in Sections 3 and 4. The operation space is divided into four domains (A to D) according to the operation boundaries of the high recycling regime and the impurity screening obtained in the present assessment. The tendency is that, if the SOL becomes thicker and the outward particle flux is enhanced due to the stochastic layer or to the island structure, the impurity screening is expected to be better. If the loss of //-momentum and the enhancement of $\perp$ energy flux become significant due to the stochastic layer or to the edge island, the high recycling regime will be lost. The direction of the optimization for future devices might be located in domain $\mathrm{D}$, where one expects both good impurity screening and high recycling regime. In domain $\mathrm{B}$, the high recycling regime might be lost. But it still can be considered as an option, if pumping efficiency is made sufficient with proper baffle/divertor design and if the detached phase is entered in order to avoid the enhanced 
physical sputtering due to a high divertor temperature expected because of the absence of high recycling regime.

The present analysis is based on a rather simplified model, neglecting some detailed features specific to each device. Nevertheless, we would like to note that the present analysis aims to obtain an overview of the transport characteristics in the 3D SOL geometry appearing in various devices. While we are aware that there is a limitation of the present model for accurate evaluation of SOL characteristics, the simplification enables us to obtain an overview of the global trend of the SOL features of each device, which is our main purpose of the present study. Certainly, more sophisticated model together with a coordinated experimental program between different devices will provide much better analysis. Such works should remain for future tasks. The present analysis is, therefore, should be considered as very rough estimation and a kind of a test to see how the simple model can work out for describing the 3D effects.

The effects on the detachment stabilization have been clearly demonstrated in Tore Supra, W7-AS and LHD by changing the edge magnetic geometry. This indicates the possibility to control the 3D edge radiation structure with a magnetic field geometry. The key parameters are the distance between the edge island (the divertor) and core plasma, $\Delta x_{L C F S-i s l a n d}$ ( $\Delta x_{\text {LCFS-div }}$ ), and the MP amplitude $B_{r} / B_{t}$. It is found that that the larger $\Delta x_{L C F S-i s l a n d}$ ( $\Delta x_{L C F S-d i v}$ ) and $B_{r} / B_{t}$ are, the better the detachment is stabilized. The results indicate that it is important to decouple the core and edge in terms of particle fuelling and to separate the radiation region from the confinement region.

In addition, with MP application there have been a variety of plasma behaviours with respect to change of electric field, turbulent transport, plasma response and particle confinement time, reported in various devices, as discussed in Section 6. The underlying mechanisms are not yet clear. It is noted that, since MP application does have clear effects on the electric field and the turbulent transport, the analysis presented in the preceding sections are affected through, $D_{\perp}, \chi_{\perp}$, used in the parameters, and ExB drift on the impurity transport etc. A comprehensive picture of the 3D effects on the SOL and divertor transport still must wait for these issues to be clarified. 


\section{References}

1 L. Spitzer, Jr. U.S. Atomic Energy Commision Report No. NYO-993 (PM-S-1), 1951.

2 L. Spitzer Jr., Physics of Fluids 1 (1958) 253.

3 K. Uo, Kakuyugou Kenkyuu 1 (1958) 20.

4 K. Uo, J. Physical Society of Japan 16 (1961) 1380.

5 P.C. Stangeby and G.M. Mccracken, Nucl. Fusion 30 (1990) 1225.

6 C.S. Pitcher and P.C. Stangeby, Plasma Phys. Control. Fusion 39 (1997) 779.

7 ITER Physics Expert Group on Divertor et al., Nucl. Fusion 39 (1999) 2391.

8 G. Federici et al., Nucl. Fusion 41 (2001) 1967.

9 A. Loarte et al., Nucl. Fusion 47 (2007) S203.

10 Kukushkin et al., Nucl. Fusion 43 (2003) 716.

11 A. Loarte, Plasma Phys. Control. Fusion 43 (2001) R183.

12 N. Ohyabu et al., Nucl. Fusion 34 (1994) 387.

13 R. Koenig et al., Plasma Phys. Control. Fusion 44 (2002) 2365.

14 E. Strumberger, Nucl. Fusion 7 (1996) 891.

15 C. Alejaldre et al., Plasma Phys. Control. Fusion 41 (1999) A539.

16 D.T. Anderson et al., Fusion Sci. Technol. 50 (2006) 171.

17 T. Mizuuchi et al., J. Nucl. Mater. 176-177 (1990) 1070.

18 T. Morisaki et al., J. Nucl. Mater. 313-316 (2003) 548.

19 Y. Feng et al., J. Nucl. Mater. 313-316 (2003) 857.

20 A. Grosman et al., Plasma Phys. Control. Fusion 32 (1990) 1011.

21 Ph. Ghendrih et al., Plasma Phys. Control. Fusion 34 (1992) 2007.

$22 \mathrm{Ph}$. Ghendrih, A. Grosman and H. Capes, Plasma Phys. Control. Fusion 38 (1996) 1653.

23 Ph. Ghendrih et al., Nucl. Fusion 42 (2002) 1221.

24 K.H. Finken et al., Nucl. Fusion 39 (1999) 637.

25 O. Schmitz et al., Nucl. Fusion 52 (2012) 043005.

26 K.H. Finken et al., “The structure of magnetic field in the TEXTOR-DED”, Scriften des Forschungszentrums Juelich, Energy Technology vol.45, Forschungszentrums Juelich GmbH (2005). ISBN: 3-89336-418-8.

27 A. Grosman et al., J. Nucl. Mater. 196-198 (1992) 59.

28 T.E. Evans et al., Nature Physics 2 (2006) 419.

29 O. Schmitz et al., J. Nucl. Mater. 438 (2013) S194.

30 T.E. Evans et al., Nucl. Fusion 53 (2013) 093029.

31 W. Suttrop et al., Phys. Rev. Lett. 106 (2011) 225004.

32 R. Fischer et al., Plasma Phys. Control. Fusion 54 (2012) 115008.

33 Y. Liang et al., Nucl. Fusion 50 (2010) 025013.

34 Y. Liang et al., Nucl. Fusion 53 (2013) 073036.

35 A. Loarte et al., Nucl. Fusion 54 (2014) 033007.

36 J-W. Ahn et al., Plasma Phys. Control. Fusion 56 (2014) 015005.

37 T.E. Evans et al., Phys. Plasmas 9 (2002) 4957.

38 T.E. Evans et al., Nucl. Fusion 48 (2008) 024002.

39 T.E. Evans et al., Phys. Plasmas 13 (2006) 056121.

40 J. Li, H.Y. Guo et al., Nature Physics 9 (2013) 817.

41 Y. Liang et al., Phys. Rev. Lett. 110 (2013) 235002.

42 M. Wischmeier et al., PSI2014, R-3.

43 B. Covele et al., Nucl. Fusion 54 (2014) 072006.

44 N. Yanagi et al., Nucl. Fusion 51 (2011) 103017.

45 N. Asakura et al., Nucl. Fusion 53 (2011) 123013.

46 Y. Feng et al., Contrib. Plasma Phys. 54 (2014) 426. 
47 D. Reiter et al., Fusion Sci. Technol. 47 (2005) 172, http://www.eirene.de/.

48 G. Ciraolo et al., Contrib. Plasma Phys. 54 (2014) 432.

49 P. Tamain et al., J. Computational Physics 229 (2010) 361.

50 C. Colin et al., PSI2014, O-24.

51 A.M. Runov et al., Phys. Plasmas 8 (2001) 916.

52 R. Zagorski et al., Nucl. Fusion 48 (2008) 024013.

53 H. Yamada et al., Fusion Sci. Technol. 58 (2010) 12.

54 O. Schmitz et al., Nucl. Fusion 48 (2008) 024009.

55 O. Schmitz et al., Plasma Phys. Control. Fusion 50 (2008) 124029.

56 M. Hirsch et al., Plasma Phys. Control. Fusion 50 (2008) 053001.

57 T.E. Evans et al., J. Physics: Conference Series 7 (2005) 174.

58 D.N. Hill and the DIII-D Team, Nucl. Fusion 53 (2013) 104001.

59 F. Romanelli and JET EFDA Contributors, Nucl. Fusion 53 (2013) 104002.

60 M. Kocan et al., Nucl. Fusion 53 (2013) 073047.

61 O. Schmitz et al., J. Nucl. Mater. 415 (2011) S886.

62 Y. Feng et al., Nucl. Fusion 49 (2009) 095002.

63 M. Kobayashi et al., Fusion Sci. Technol. 58 (2010) 220.

64 H. Frerichs et al., Nucl. Fusion 52 (2012) 054008.

65 T. Lunt et al, Nucl. Fusion 52 (2012) 054013.

66 T. Lunt et al, J. Nucl. Mater. 438 (2013) S254.

67 D.W. Kerst, Plasma Physics (J. Nuclear Energy, Part C) 4, 253 (1962).

68 M.N. Rosenbluth et al., Nucl. Fusion, 6, 297 (1966).

69 F. Nguyen et al., Nucl. Fusion 37 (1997) 743.

70 K. Ida et al., New Journal of Physics 15 (2013) 013061.

71 K. Ida et al., “ Evidence of Stochastic Region near a Rational Surface in Core Plasmas of LHD”, 23rd IAEA FEC (2010), EXS/5-2, Daejeon, Korea.

72 K. Itoh, S-I. Itoh and A. Fukuyama, Transport and structural formation in plasmas, London: Institute of Physics Publishing, 1999, pp. 36.

73 B.B. Kadomtsev and O.P. Pogutse, Plasma Physics and Controlled Nuclear Fusion Research, Proceedings, 7th International Conference, Innsbruck, 1978 (International Atomic Energy Agency, Vienna, 1978) vol. 1, p.649.

74 C.W. Horton, Hand Book of Plasma Physics, edited by M.N. Rosenbluth and R.E. Sagleev, Amsterdam: Elsevier Science Publishers B.V., 1984, vol. 2, Section 6.4, p.435.

75 I. Garcia-Cortes et al., Fusion Sci. Technol. 50 (2006) 307.

76 Y. Feng et al., Plasma Phys. Control. Fusion 53 (2011) 024009.

77 F.L. Hinton and R.D. Hazeltine, Reviews of Modern Physics 48 (1976) 239.

78 S.P. Hirshman and D.J. Sigmar, Nucl. Fusion 21 (1981) 1079.

79 K.C. Shaing, S.P. Hirshman and J.D. Callen, Phys. Fluids 29 (1986) 521.

80 K. Ida and N. Nakajima, Phys. Plasmas 4 (1997) 310.

81 B. Chatthong and T. Onjun, Nucl. Fusion 53 (2013) 013007.

82 H. Frerichs et al., Phys. Plasmas 21 (2014) 020702.

83 P. C. Stangeby, “The Plasma Boundary of Magnetic Fusion Devices”, Institute of Physics Publishing (2000).

84 M.A. Mahdavi et al., Phys. Rev. Lett. 47 (1981) 1602.

85 Y. Shimomura et al., Nucl. Fusion 23 (1983) 869.

86 J. Neuhauser et al., Plasma Phys. Control. Fusion 31 (1989) 1551.

87 A. Loarte et al., Nucl. Fusion 38 (1998) 331.

88 B. Lipschultz et al., J. Nucl. Mater. 220-222 (1995) 50.

89 B. Lipschultz et al., Fusion Science and Technology 51 (2007) 369. 
90 N. Asakura et al., Nucl. Fusion 35 (1995) 381.

91 M. Groth et al., J. Nucl. Mater. 415 (2011) S530.

92 P.C. Stangeby, Nucl. Fusion 33 (1993) 1695.

93 B. LaBombard et al., J. Nucl. Mater. 241-243 (1997) 149.

94 B. LaBombard et al., Phys. Plasmas 2 (1995) 2242.

95 P. Grigull et al., Plasma Phys. Control. Fusion 43 (2001) A175.

96 Y. Feng et al., Plasma Phys. Control. Fusion 44 (2002) 611.

97 Y. Feng et al., Nucl. Fusion 48 (2008) 024012.

98 S. Masuzaki et al., J. Nucl. Mater. 313-316 (2003) 852.

99 M. Clever et al., Nucl. Fusion 52 (2012) 054005.

100 Y. Feng et al., Contrib. Plasma Phys. 44 (2004) 57.

101 N. Ezumi et al., J. Plasma Fusion Res. SERIES 8 (2009) 429.

102 M. Kobayashi et al., J. Plasma Fusion Res. 85 (2009) 393, in Japanese.

103 A. Bader et al., Nucl. Fusion 53 (2013) 113036.

104 Y. Feng et al., Nucl. Fusion 46 (2006) 807.

105 H. Frerichs et al., Nucl. Fusion 52 (2012) 023001.

106 M.Z. Tokar et al., Phys. Plasmas 11 (2004) 4610.

107 A. Loarte J. Nucl. Mater. 241-243 (1997) 118.

108 D.P. Stotler et al., J. Nucl. Mater. 266-269 (1999) 947.

109 B. Meslin et al., J. Nucl. Mater. 266-269 (1999) 318.

110 M. Lehnen et al., J. Nucl. Mater. 337-339 (2005) 171.

111 Y. Corre et al., Nucl. Fusion 47 (2007) 119.

112 H. Frerichs et al., submitted to Physics of Plasmas.

113 Y. Corre, PhD Thesis, L'UNIVERSTITE PARIS VI, (2001) EUR-CEA-FC-1722.

114 H. Frerichs et al., Nucl. Fusion 50 (2010) 034004.

115 H. Renner et al., Nucl. Fusion 40 (2000) 1083.

116 O. Schmitz et al., O-10, International Stellarator/Heliotron Workshop, 2013 Sep. 16-20, Padua, Italy.

117 H. Frerichs, private communication

118 A. Loarte, Contrib. Plasma Phys. 32 (1992) 468.

119 C.J. Lasnier et al., Nucl. Fusion 38 (1998) 1225.

120 K. Hoshino et al., J. Nucl. Mater. 415 (2011) S549.

121 T. Eich et al., Nucl. Fusion 53 (2013) 093031.

122 J.R. Harrison et al., PSI2014 O-25.

123 S. Lippmann et al., Nucl. Fusion 31 (1991) 2131.

124 C. Breton et al., Nucl. Fusion 31 (1991) 1774.

125 G. Telesca et al., J. Nucl. Mater. 390-391 (2009) 227.

126 M. Lehnen et al., Plasma Phys. Control. Fusion 47 (2005) B237.

127 M. Kobayashi et al., Nucl. Fusion 53 (2013) 033011.

128 S. Morita et al., Nucl. Fusion 53 (2013) 093017.

129 Y. Nakamura et al., Nucl. Fusion 56 (2014) 075014.

130 Y. Nakamura et al., Plasma Phys. Control. Fusion 44 (2002) 2121.

131 Y. Nakamura et al., Nucl. Fusion 43 (2003) 219.

132 M.B. Chowdhuri et al., Phys. Plasmas 16 (2009) 062502.

133 K. Ida et al., Phys. Plasmas 16 (2009) 056111.

134 M. Yoshinuma et al., Nucl. Fusion 49 (2009) 062002.

135 R. Burhenn et al., Fusion Science \& Technology 53 (2004) 115.

136 H. Zhou et al., Jpn. J. Appl. Phys. 49 (2010) 106103.

137 R. Dei-cas and A. Samain, Plasma Phys. Control. Nucl. Fusion Res. 1 (1975) 563. 
138 A.A. Shishkin, Nucl. Fusion 21 (1981) 603.

139 A. Samain, A. Grosman and W. Feneberg, J. Nucl. Mater.111-112 (1982) 408.

140 A. Samain et al, J. Nucl. Mater. 128-129 (1984) 395.

141 A. Nicolai, F. Schoengen and D. Reiter, Plasma Phys. 27 (1985) 1479.

142 M.Z. Tokar et al., Plasma Phys. Control. Fusion 39 (1997) 569.

143 M. Kobayashi et al., J. Nucl. Mater. In press.

144 I. Garcia-Cortes et al., J. Nucl. Mater. 337-339 (2005) 441.

145 S. Ishida et al., Nucl. Fusion 39 (1999) 1211.

146 H.Y. Guo et al., Nucl. Fusion 40 (2000) 379.

147 O. Gruber et al., Nucl. Fusion 39 (1999) 1321.

148 S. Brezinsek et al., PSI2014, R-2.

149 A. Kallenbach et al., Nucl. Fusion 49 (2009) 045007.

150 J.D. Strachan et al., J. Nucl. Mater. 337-339 (2005) 25.

151 J.D. Strachan et al., Nucl. Fusion 43 (2003) 922.

152 J.D. Strachan et al., Nucl. Fusion 44 (2004) 772.

153 H. Zhou et al., Jpn. J. Appl. Phys. 49 (2010) 106103.

154 T.E. Evans et al., J. Nucl. Mater. 196-198 (1992) 421.

155 P. Grigull et al., J. Nucl. Mater. 313-316 (2003) 1287.

156 Y. Feng et al., Nucl. Fusion 45 (2005) 89.

157 M.Z. Tokar et al., J. Nucl. Mater. 266-269 (1999) 958.

158 H. Kastelewicz et al., Plasma Phys. Control. Fusion 37 (1995) 723.

159 M. Kobayashi et al., Nucl. Fusion 53 (2013) 093032.

160 M. Kobayashi et al., Phys. Plasmas 17 (2010) 056111.

161 E.A. Drapiko et al., Nucl. Fusion 51 (2011) 073005.

162 B.J. Peterson et al., Plasma and Fusion Research 8 (2013) 2402037.

163 M. Kobayashi et al., " Effects of magnetic field structure of edge stochastic layer on radiative divertor operation in LHD”, Joint 19th ISHW and 16th IEA - RFP workshop 16 - 20 September 2013, Padova, Italy.

164 Y. Liang et al., Phys. Rev. Lett. 94 (2005) 105003.

165 Y. Xu et al., Nucl. Fusion 47 (2007) 1696.

166 O. Schmitz et al., J. Nucl. Mater. 390-391 (2009) 330.

167 J.W. Coenen et al., Nucl. Fusion 51 (2011) 063030.

168 J. Payan et al., Nucl. Fusion 35 (1995) 1357.

169 P. Devynck et al., Nucl. Fusion 42 (2002) 697.

170 S.C. McCool et al., Nucl. Fusion 29 (1989) 547.

171 A.J. Wootton, J. Nucl. Mater. 176-177 (1990) 77.

172 S. Takamura et al., Nucl. Fusion 28 (1988) 183.

173 P. Tamain et al., Plasma Phys. Control. Fusion 52 (2010) 075017.

174 J.R. Robinson et al., Plasma Phys. Control. Fusion 54 (2012) 105007.

175 H. W. Muller et al, J. Nucl. Mater. 438 (2013) S64-S71.

176 G.D.Conway et al., Plasma Phys. Control. Fusion 57 (2015) 014035.

177 J. A. Boedo et al., "Plasma Response to Applied Resonant Magnetic Perturbations in DIII-D”, 38th EPS Conference on Plasma Physics (2011), P1.122.

178 H. Tanaka et al., Phys. Plasmas 17 (2010) 102509.

179 P. Scarin et al., 23rd IAEA FEC (2010), Daejeon, Korea.

180 Y. Xu et al., Nucl. Fusion 49 (2009) 035005.

181 D. Reiser, Phys. Plasmas 14 (2007) 082314.

182 O.E. Garcia et al., Phys. Plasmas 13 (2006) 082309.

183 Y. Xu et al., Nucl. Fusion 51 (2011) 063020. 
184 P. Beyer et al., Plasma Phys. Control. Fusion 44 (2002) 2167.

185 M. Leconte and P.H. Diamond, Phys. Plasmas 18 (2011) 082309.

186 M. Leconte, P.H. Diamond and Y. Xu, Nucl. Fusion 54 (2014) 013004.

187 A.W. Leonard et al., Phys. Rev. Lett. 78 (1997) 4769.

188 A.G. McLean et al. PSI2014 O-26. 
Table 1. Parameter range for each device as used for the analysis.

\begin{tabular}{|c|c|c|c|c|c|c|c|c|c|c|}
\hline & HSX & TJ-II & DIII-D & TEXTOR-DED & EAST & W7-AS & Tore Supra & LHD & W7-X & ITER \\
\hline $\mathrm{R}(\mathrm{m})$ & $\sim 1.2(1.0-1.4)$ & 1.5 & 1.67 & 1.75 & 1.85 & 2 & 2.38 & 3.9 & 5.5 & 6.2 \\
\hline$a(\mathrm{~m})$ & $\sim 0.15(0.1-0.3)$ & $\sim 0.17$ & $0.60-0.67$ & $\sim 0.46$ & $\sim 0.45$ & $\sim 0.16$ & $\sim 0.8$ & $\sim 0.7$ & $\begin{array}{c}\sim 0.55 \\
\end{array}$ & $\sim 2.0$ \\
\hline$\lambda_{\mathrm{st}, \mathrm{SOL}}(\mathrm{m})$ & $0.005-0.07$ & $\sim 0.01\left(\sim 0.03^{*}\right)$ & $\begin{array}{c}0.05-0.10 \\
\text { (outboard midplane) }\end{array}$ & $\begin{array}{c}\sim 0.05(\mathrm{~m} / \mathrm{n}=12 / 4) \\
\sim 0.10(6 / 2) \\
\sim 0.20(3 / 1) \\
\end{array}$ & $\sim 0.02$ & $\sim 0.04$ & $\begin{array}{c}\sim 0.16 \\
(20 \% \text { of } a)\end{array}$ & $\sim 0.1$ & $\sim 0.1$ & $\sim 0.2(0.08-0.7)$ \\
\hline $\begin{array}{l}\text { Mode number of MP } \\
(\mathrm{m} / \mathrm{n})\end{array}$ & none & none & $\begin{array}{c}\sim 10 / 3,(\mathrm{~m}=3 \sim 15, \\
\mathrm{n}=1 \sim 3)\end{array}$ & $12 / 4,6 / 2,3 / 1$ & $\sim 3 / 1^{*}$ & none & $\begin{array}{c}\text { 18/6, } \\
(\mathrm{m}=18 \pm 3)\end{array}$ & $1 / 1$ & none & $\begin{array}{c}\sim 10 / 3(\mathrm{n}=3,4 \\
\mathrm{m}=6-14)\end{array}$ \\
\hline $\begin{array}{l}\text { Mode number (main } \\
\text { edge structure, } \mathrm{m} / \mathrm{n} \text { ) }\end{array}$ & $7 / 8,(4 / 4)^{*}$ & $2 / 4$ & Axi-sym. & Axi-sym. & Axi-sym. & $9 / 5$ & Axi-sym. & $2 \sim 5 / 10$ & $\begin{array}{c}(6 / 5), 5 / 5 \\
(4 / 5)\end{array}$ & Axi-sym. \\
\hline$q_{\text {edge }} @$ LCFS & $0.82-1.0$ & 0.5 & $\sim 3.5(3.0-4.2)$ & 3.6 & $3.8-4.7$ & 1.8 & $\sim 3$ & 1 & $(0.8), 1.0,(1.2)$ & 3.2, (4.3) \\
\hline$L_{K}(\mathrm{~m})$ & & & $125-220$ & $\begin{array}{c}\sim 70\left(\mathrm{~m} / \mathrm{n}=12 / 4, \mathrm{I}_{\mathrm{DED}}=7 \mathrm{kA}\right), \\
\sim 50(12 / 4,15 \mathrm{kA}), \\
20-35(6 / 2,7.5 \mathrm{kA})\end{array}$ & & & $\sim 10$ & $10-100$ & $37-57$ & \\
\hline $\mathrm{B}_{\mathrm{r}}\left(10^{-3} \mathrm{~T}\right)$ & $(0.22-0.58)$ & $(5.6 *)$ & $0.14-0.39$ & $\sim 1$ & $\sim 0.5$ & $\sim 2.5$ & $3-4.65$ & $0.264-2.64$ & $\sim 3$ & $(\sim 2.7)$ \\
\hline $\mathrm{B}_{\mathrm{T}}(\mathrm{T})$ & 1 & 0.96 & $1.65-2.10$ & $\sim 2$ & 3.5 & $\sim 2.5$ & $\sim 3$ & 2.64 & $\sim 3$ & 5.3 \\
\hline $\mathrm{B}_{\mathrm{r}} / \mathrm{B}_{\mathrm{T}}$ & $2.2-5.8 \times 10^{-4}$ & $5.8 \times 10^{-3 *}$ & $\begin{array}{c}5.1-8.4 \times 10^{-4} \text { (total), } \\
1.4-3.9 \times 10^{-4}(\mathrm{~m} / \mathrm{n}=11 / 3)\end{array}$ & $\sim 5 \times 10^{-4}$ & $\sim 1.4 \times 10^{-4}$ & $10^{-3}$ & $\begin{array}{c}1.0 \sim 1.5 \mathrm{x} \\
10^{-3} \\
\end{array}$ & $10^{-4}-10^{-3}$ & $10^{-3}$ & $\sim 5 \times 10^{-4}$ \\
\hline $\mathrm{T}_{\mathrm{e}, \text { LCFS }}(\mathrm{eV})$ & $200 * *$ & $30-50$ & $60-90$ & $40-100$ & $30-80$ & $20-150$ & $100-300$ & $150-300$ & $160-200$ & $160-200 *$ \\
\hline $\mathrm{n}_{\mathrm{e}, \text { LCFS }}\left(10^{19} \mathrm{~m}^{-3}\right)$ & $2-12 * *$ & $0.1-0.2$ & $0.5-1.5$ & $1-5$ & $0.3-0.6$ & $1-6$ & $1.0-1.8$ & $1-6$ & $1.5-3$ & $3-5^{*}$ \\
\hline$D_{\perp}\left(\mathrm{m}^{2} / \mathrm{s}\right)$ & 1.0 & 1.0 & 0.2 & 1.0 & $(0.5)$ & 0.5 & 1.0 & 0.5 & 0.5 & 0.4 \\
\hline Remark & $\begin{array}{l}\text { * For large island } \\
\text { configuration } \\
\text { ** numerical } \\
\text { simulation }\end{array}$ & $\begin{array}{l}* \text { For island } \\
\text { divertor type } \\
\text { configuration }\end{array}$ & $\begin{array}{l}\text { Lower triangularity } \\
\quad(0.26-0.65)\end{array}$ & & $\begin{array}{c}* \text { Current } \\
\text { filaments } \\
\text { induced by } \\
\text { LHCD } \\
\end{array}$ & & & & & $\begin{array}{l}\text { *According to } \\
\text { axi-symmetric } \\
\text { configuration }\end{array}$ \\
\hline References & 16,103 & $15,75,144$ & 25,28,37,38,39 & 24,25,26,54,,105 & 40,41 & 19,95 & $\begin{array}{c}20,21,22,23 \\
27,69\end{array}$ & $12,18,98,127$ & 14,115 & 10,29 \\
\hline
\end{tabular}




\section{Table 2}

Table 2. Summary of the 3D effects on the SOL and divertor transport in various devices. $\dagger$ : Numerical simulations. The numbers at the devices represent the references of the observations. The definitions of the formulae of key parameters are given in the text.

\begin{tabular}{|c|c|c|c|c|}
\hline Observation & Devices & Key parameters & Interpretation & Impact on divertor function \\
\hline$n_{\text {down }} \propto n_{\text {up }}^{\alpha}$ & \multirow{3}{*}{$\begin{array}{l}\text { W7-AS }{ }^{104}, \text { LHD }^{63} \\
\text { TEXTOR-DED } \\
\text { HSX,105,106, } \\
\text { HS } 103\end{array}$} & $\tau_{m / /} / \tau_{m \perp}>1$ & $\begin{array}{l}\text { Loss of //-momentum } \\
\qquad \rightarrow p_{\text {nn }}>p_{d}\end{array}$ & \multirow{3}{*}{$\begin{array}{c}\text { Pumping efficiency } \downarrow \\
\text { Phys. Sputtering } \uparrow \\
\text { Detach. onset density } \uparrow \text { (?) }\end{array}$} \\
\hline$T_{\text {down }} \propto n_{\text {up }}{ }^{-\alpha+1}$ & & & Fup F Fown & \\
\hline $\begin{array}{l}\alpha=3 \rightarrow \leq 2 \\
\text { (Absence of high } \\
\text { recycling regime) }\end{array}$ & & $\begin{array}{l}q_{\perp e} / q_{/ / e}>>1 \\
q_{/ / \text {conv }} / q_{/ / \text {cond }} \uparrow\end{array}$ & Reduction of //-energy conduction & \\
\hline \multirow{3}{*}{ Core decontamination } & \multirow{3}{*}{ 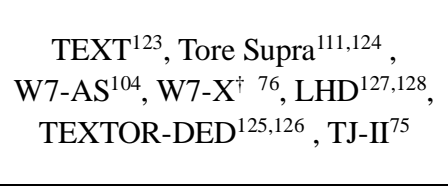 } & $\Gamma_{s t}^{p} / \Gamma_{\perp}^{p}>>1$ & Enhanced outward particle flux (friction force) & \multirow{3}{*}{ Impurity screening $\uparrow$} \\
\hline & & $q_{\perp i} / q_{/ / i}>>1$ & Ion thermal force suppression & \\
\hline & & $\lambda_{\text {st-SOL }} / \lambda_{\text {imp }} \uparrow$ & Thicker SOL/stochastic layer & \\
\hline \multirow[b]{2}{*}{ Detach. stabilization } & \multirow[b]{2}{*}{$\begin{array}{l}\text { W7-AS }{ }^{155,156}, \text { LHD }^{159} \\
\text { Tore Supra }^{154}\end{array}$} & $\tilde{B}_{r} / B_{t}\left(w_{\text {island }}\right) \uparrow$ & Radiation modulation by islands & \multirow[b]{2}{*}{ Heat removal $\uparrow$} \\
\hline & & $\begin{array}{l}\Delta x_{\text {LCFS-island }} \uparrow \\
\left(\Delta x_{\text {LCFS-div }}\right)\end{array}$ & $\begin{array}{c}\text { Core edge decoupling } \\
\rightarrow \text { Particle fueling } \downarrow \\
\rightarrow \text { Core impurity penetration } \downarrow \\
\end{array}$ & \\
\hline $\begin{array}{c}\text { MARFE onset } \\
\text { delayed }\end{array}$ & TEXTOR-DED ${ }^{164}$ & $f_{R M P}$ & $\begin{array}{l}\text { Avoid localized cooling by spreading recycling } \\
\text { region with MP rotation }\end{array}$ & Density limit $\uparrow(?)$ \\
\hline
\end{tabular}




\section{Figures captions}

Fig.1 Schematics of divertor configurations and transport directions for (a) Limiter + MP (type I), (b) Island divertor (type II) and (c) X-point poloidal divertor + MP, helical divertor (type III), respectively. $\Gamma_{/ /, \perp}$ represent fluxes of physical quantities (particle, momentum, energy) that are parallel and perpendicular the magnetic field lines, respectively. Subscripts $r$ and $\theta$ indicate a radial and poloidal components of the fluxes, respectively.

Fig.2 Schematics of formation of //-flow towards divertor plates in (a) 2D axi-symmetric and in (b) 3D divertor configurations. $+/-\phi$ and $+/-V_{/ /}$represent positive and negative directions of toroidal angle and of corresponding //-plasma flows, respectively. $\lambda_{m}$ represents the spatial separation between counter streaming flows $\left(+/-V_{/ /}\right)$. In the 2D case, the flux tubes are connected to inner and outer divertor plates in directions toroidally opposite $(+/-\phi)$ each other. A similar situation takes places at the flux tubes in the 3D case, where the magnetic island structure is opened and connected to the divertor plates. In the $3 \mathrm{D}$ case, $\lambda_{m}$ becomes short and thus momentum loss of //-flow via $\perp$-viscosity takes place.

Fig. 3 Schematics of deformation of flux tubes in the stochastic layers. Through bending of flux tubes by $B_{r}$ and stretching by magnetic shear, under a flux conservation, the flux tubes of long and short $\mathrm{L}_{C}$ are strongly deformed. The resultant interaction area increases and thus $\perp$ exchange of plasma quantities is enhanced with substantial plasma density causing viscosity.

Fig.4 (a) Divertor density $\left(n_{\text {down }}\right)$ and (b) temperature $\left(T_{\text {down }}\right)$ as a function of upstream density ( $n_{\text {up }}$ ) for LHD [98], W7-AS [96] and TEXTOR-DED (m/n=6/2) [99]. The 3D divertor configurations show modest density dependence, $n_{\text {down }} \propto n_{\text {up }}{ }^{\sim 1}, T_{\text {down }} \propto n_{\text {up }}{ }^{-\sim 1}$, compared to the high recycling regime in 2D tokamaks, $n_{\text {down }} \propto n_{\text {up }}{ }^{3}, T_{\text {down }} \propto n_{\text {up }}{ }^{-2}$. 
Fig. 5 Dependence of (a) $n_{\text {down }}$ (b) $T_{\text {down }}$ and $T_{u p}$ on upstream density $n_{u p}$, including $\perp$ loss of //-momentum, obtained by extended two point model [104]. Blue curves: 2D axi-symmetric divertor red curves: 3D divertor. Dashed lines in (b): $T_{u p}$ and solid lines : $n_{\text {down }}$ and $T_{\text {down }}$. By increasing the momentum loss factor, $f_{m 0}$, the upstream and downstream coupling becomes weak and the density dependence deviates from the high-recycling regime in a 2D axi-symmetric divertor.

Fig.6 (a) 2D distribution of //-particle flux ( $\left.\mathrm{nV}_{/ /}\right)$in a poloidal cross section obtained by 3D simulation (EMC3-EIRENE). Bright colors (yellow and red) and dark colors (blue and black) represent //-flows in positive and negative toroidal directions, respectively. (b) Vertical profiles of Mach number measured by scanning Mach probe (symbols) together with the results of 3D simulations (solid line) [101]. The scanning path of the Mach probe is indicated in (a).

Fig. 7 Divertor density regime of various devices in terms of $\perp$ loss of //-momentum, $\tau_{m / /} / \tau_{m \perp}$, and replacement of //-energy flux with $\perp$-flux, $q_{\perp e} / q_{/ / e}$. Red: no high recycling regime $(\alpha<3)$, blue: with high recycling regime $(\alpha \approx 3)$, where $n_{\text {down }} \propto n_{\text {up }}{ }^{\alpha}$. The results from numerical simulations are plotted with dashed lines. The larger the $\tau_{m / /} / \tau_{m \perp}$ or $q_{\perp e} / q_{/ / e}$ are, the weaker the upstream and downstream coupling, i.e., density dependence, are. The shaded area indicates the operation domain given by $\left(\frac{\tau_{m / /}}{\tau_{m \perp}}\right)^{2}\left(\frac{q_{\perp e}}{q_{/ / e}}\right)<3.6 \times 10^{-5}$.

Fig.8 Mach number distribution in the ITER with MP application, obtained by 3D numerical simulation (EMC3-EIRENE) [29]. Red and blue colors represent //-flows in positive and negative toroidal directions, respectively. Counter streaming flows appear.

Fig. 9 Density dependence of carbon content in plasma for (a) Tore Supra [111], (b) TEXTOR-DED (m/n=3/1) [126] and (c) LHD.

Fig.10 Density dependence of the carbon emission ratio (CV+CVI) to (CIII+CIV) for thin (diamonds) and thick (circles) stochastic layer widths in LHD [132]. The ratio is 
interpreted as the ratio of the carbon content in core plasma to the source, as discussed in the text.

Fig.11 Operating domain of various devices in the space of (a) $\frac{\lambda_{s t-S O L}}{\lambda_{\text {imp }}}$ and $\frac{\Gamma_{s t}^{p}}{\Gamma_{\perp}^{p}}$, and (b) $\frac{\lambda_{s t-S O L}}{\lambda_{\text {imp }}}$ and $\frac{q_{\perp i}}{q_{/ / i}}$. The observation of impurity screening is indicated with blue circles, and the observation of no impurity screening with red circles. The results from numerical simulations are plotted with dashed lines. $\frac{\lambda_{s t-S O L}}{\lambda_{\text {imp }}}$ is the ratio of the radial width of the stochastic layer or island divertor SOL to the neutral impurity penetration length for carbon. $\frac{\Gamma_{s t}^{p}}{\Gamma_{\perp}^{p}}$ and $\frac{q_{\perp i}}{q_{/ / i}}$ represent the enhancement of the radially outward particle flux and of $\perp$-ion energy flux, respectively. The operating domain of the upper half of the density range is plotted for all devices, taking into account the fact that the screening effects are pronounced always at high density operation. The results of numerical simulation are indicated with dashed lines. The shaded area in (a) indicates the operating domain given by $\left(\frac{\lambda_{s t-S O L}}{\lambda_{\text {imp }}}\right)\left(\frac{\Gamma_{s t}^{p}}{\Gamma_{\perp}^{p}}\right)^{1 / 4}>18$.

Fig.12 Carbon radiation distribution in W7-AS for (a) stable detachment with large $\Delta x_{L C F S-d i v}$ and for (b) unstable detachment with small $\Delta x_{L C F S-d i v}$, obtained by numerical simulations (EMC3-EIRENE). [156]

Fig.13 Carbon radiation distribution in LHD for (a) stable detachment with MP $(\mathrm{m} / \mathrm{n}=1 / 1)$ application and for (b) unstable detachment without MP, obtained by numerical simulations (EMC3-EIRENE). The line integrated radiation profile measurements along the line of sight as indicated with white lines, are plotted together on the right column, in comparison with the simulations [159].

Fig. 14 Operation domain of the stable detachment in LHD and W7-AS in terms of 
$\Delta x_{\text {LCFS-island }}\left(\Delta x_{L C F S-d i v}\right)$ and $B_{r} / B_{t}$. The definitions of $\Delta x_{L C F S-i s l a n d}$ and $\Delta x_{\text {LCFS }- \text { div }}$ are given in the right figures.

Fig.15 Schematic of possible optimization of 3D divertor configuration in terms of $\left(\frac{\tau_{m / /}}{\tau_{m \perp}}\right)^{2}\left(\frac{q_{\perp e}}{q_{/ / e}}\right)$ (as a measure of enhancement of $\perp$-energy and momentum transport) and of $\left(\frac{\lambda_{s t-S O L}}{\lambda_{\text {imp }}}\right)^{2}\left(\frac{\Gamma_{s t}^{p}}{\Gamma_{\perp}^{p}}\right)^{1 / 4}$ (as a measure of SOL thickness and of enhanced outward particle flux). The operation space is divided into four domains (A to D) according to the operation boundaries of the high recycling regime and of impurity screening.

Fig.A1 Operating domain of each device in the space of (a) $\tau_{m / /} / \tau_{m \perp}-\lambda_{s t-S O L} / \lambda_{i m p}$ and (b) $\left(\delta r / \lambda_{m}\right)^{2}-\lambda_{\text {st-SOL }} / \lambda_{\text {imp }}$. 


\section{Figures}

Fig.1

(a)

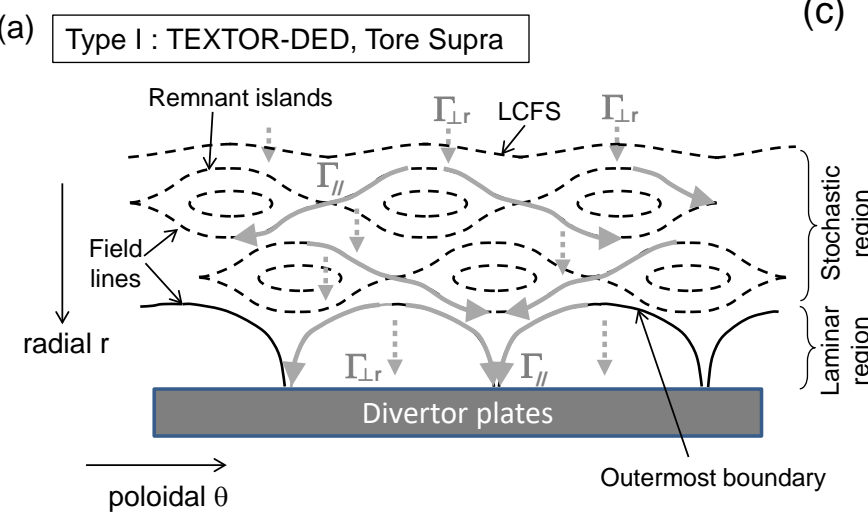

(c) Type III : DIII-D, EAST, LHD, ITER

(b) Type II : W7-AS/X, HSX, TJ-II

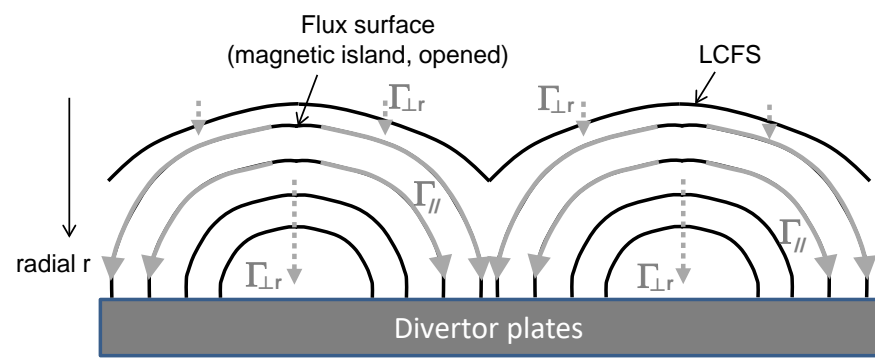

Poloidal $\theta$

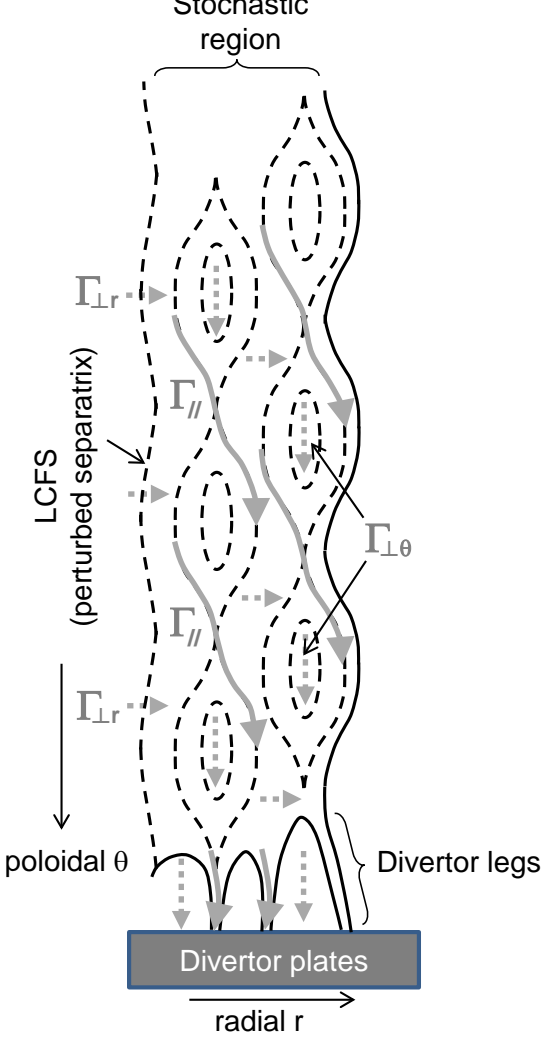

Fig.1 Schematics of divertor configurations and transport directions for (a) Limiter + MP (type I), (b) Island divertor (type II) and (c) X-point poloidal divertor + MP, helical divertor (type III), respectively. $\Gamma_{/ /, \perp}$ represent fluxes of physical quantities (particle, momentum, energy) that are parallel and perpendicular the magnetic field lines, respectively. Subscripts $r$ and $\theta$ indicate a radial and poloidal components of the fluxes, respectively. 
Fig.2

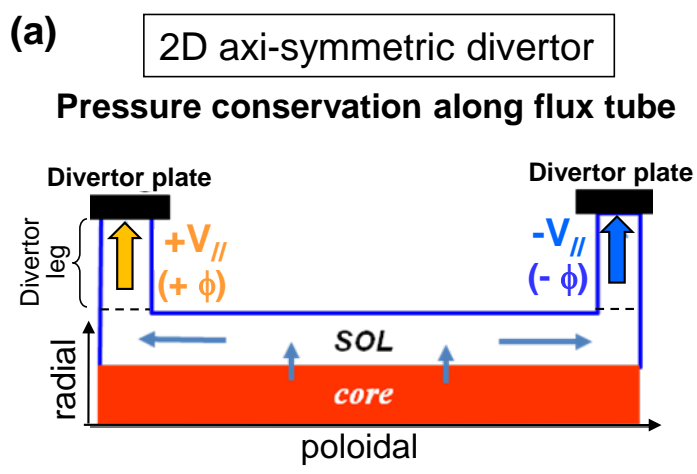

(b)

3D configuration (e.g. stochastic layer, ID)

II-Momentum loss due to counter flows

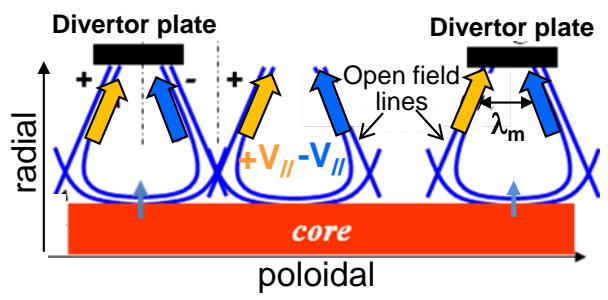

Fig.2 Schematics of formation of //-flow towards divertor plates in (a) 2D axi-symmetric and in (b) 3D divertor configurations. $+/-\phi$ and $+/-V_{/ /}$represent positive and negative directions of toroidal angle and of corresponding //-plasma flows, respectively. $\lambda_{m}$ represents the spatial separation between counter streaming flows $\left(+/-\mathrm{V}_{/ /}\right)$. In the 2D case, the flux tubes are connected to inner and outer divertor plates in directions toroidally opposite $(+/-\phi)$ each other. A similar situation takes places at the flux tubes in the 3D case, where the magnetic island structure is opened and connected to the divertor plates. In the $3 \mathrm{D}$ case, $\lambda_{m}$ becomes short and thus momentum loss of //-flow via $\perp$-viscosity takes place. 
Fig.3

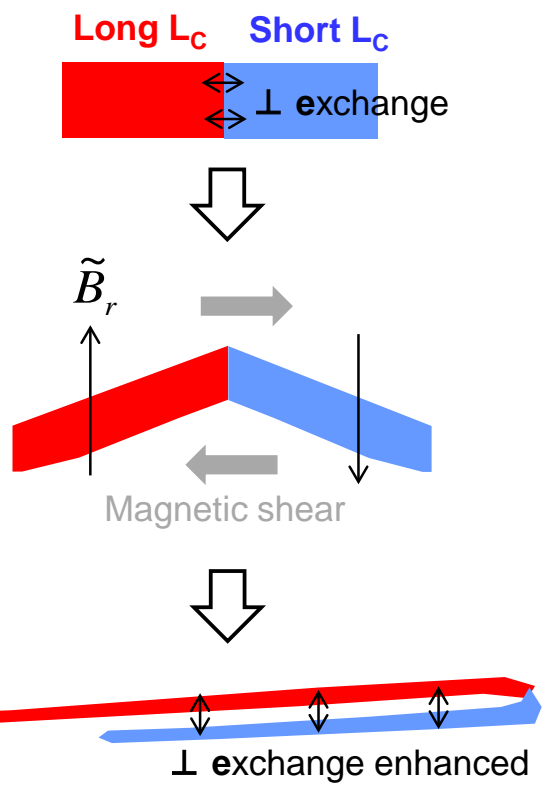

Fig. 3 Schematics of deformation of flux tubes in the stochastic layers. Through bending of flux tubes by $B_{r}$ and stretching by magnetic shear, under a flux conservation, the flux tubes of long and short $\mathrm{L}_{\mathrm{C}}$ are strongly deformed. The resultant interaction area increases and thus $\perp$ exchange of plasma quantities is enhanced with substantial plasma density causing viscosity. 
Fig.4
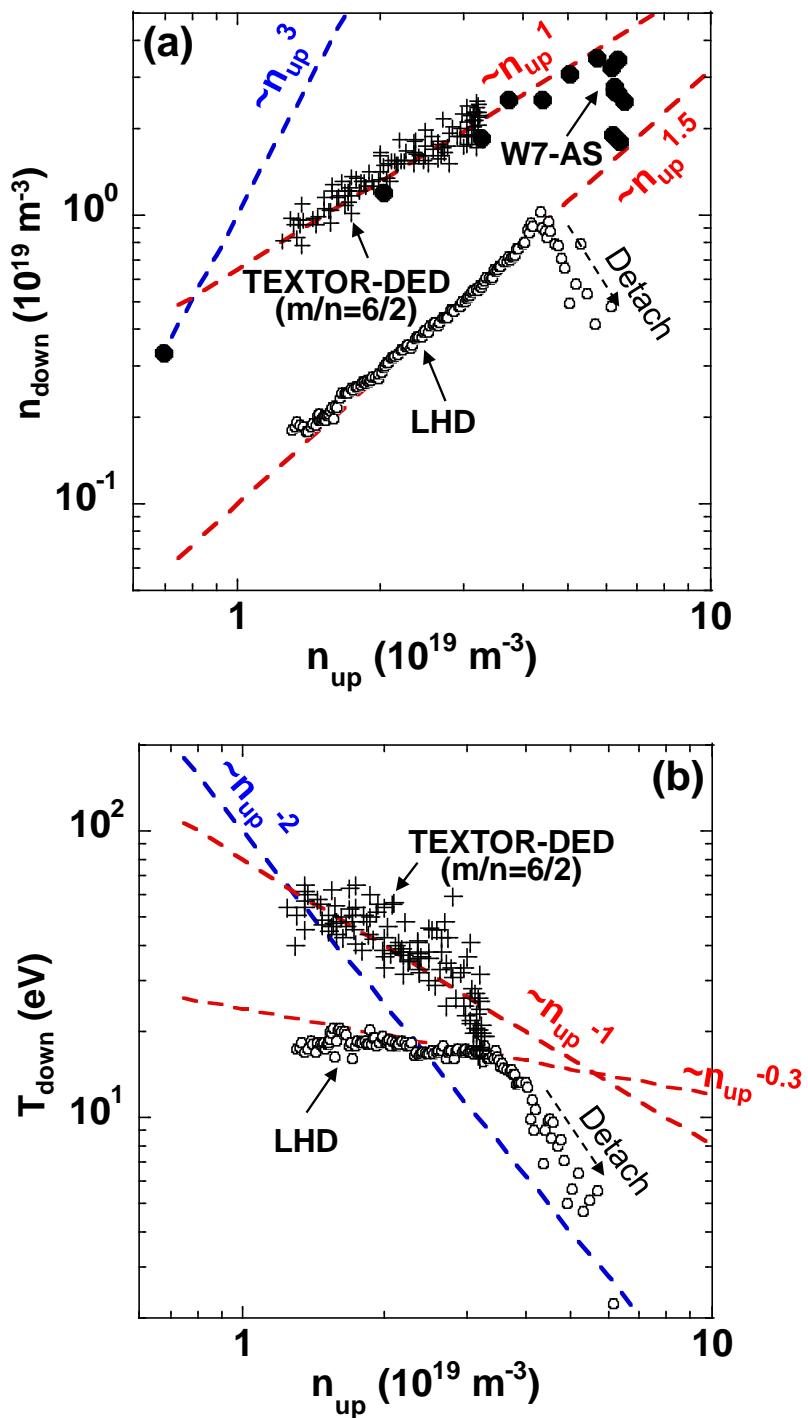

Fig.4 (a) Divertor density $\left(n_{\text {down }}\right)$ and (b) temperature $\left(T_{\text {down }}\right)$ as a function of upstream density ( $n_{u p}$ ) for LHD [98], W7-AS [96] and TEXTOR-DED (m/n=6/2) [99]. The 3D divertor configurations show modest density dependence, $n_{\text {down }} \propto n_{\text {up }}{ }^{\sim 1}, T_{\text {down }} \propto n_{\text {up }}{ }^{-\sim 1}$, compared to the high recycling regime in 2D tokamaks, $n_{\text {down }} \propto n_{\text {up }}{ }^{3}, T_{\text {down }} \propto n_{\text {up }}{ }^{-2}$. 
Fig.5
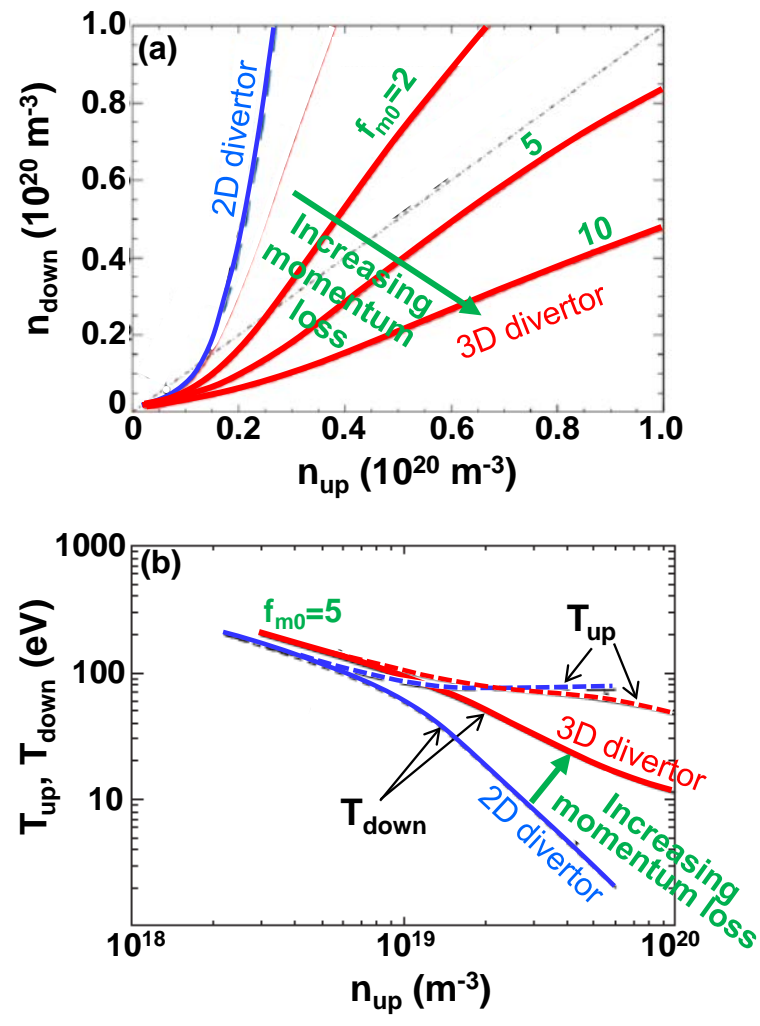

Fig. 5 Dependence of (a) $n_{\text {down }}$ (b) $T_{\text {down }}$ and $T_{u p}$ on upstream density $n_{\text {up }}$, including $\perp$ loss of //-momentum, obtained by extended two point model [104]. Blue curves: 2D axi-symmetric divertor red curves: 3D divertor. Dashed lines in (b): $T_{u p}$ and solid lines : $n_{\text {down }}$ and $T_{\text {down }}$. By increasing the momentum loss factor, $f_{m 0}$, the upstream and downstream coupling becomes weak and the density dependence deviates from the high-recycling regime in a 2D axi-symmetric divertor. 
Fig.6
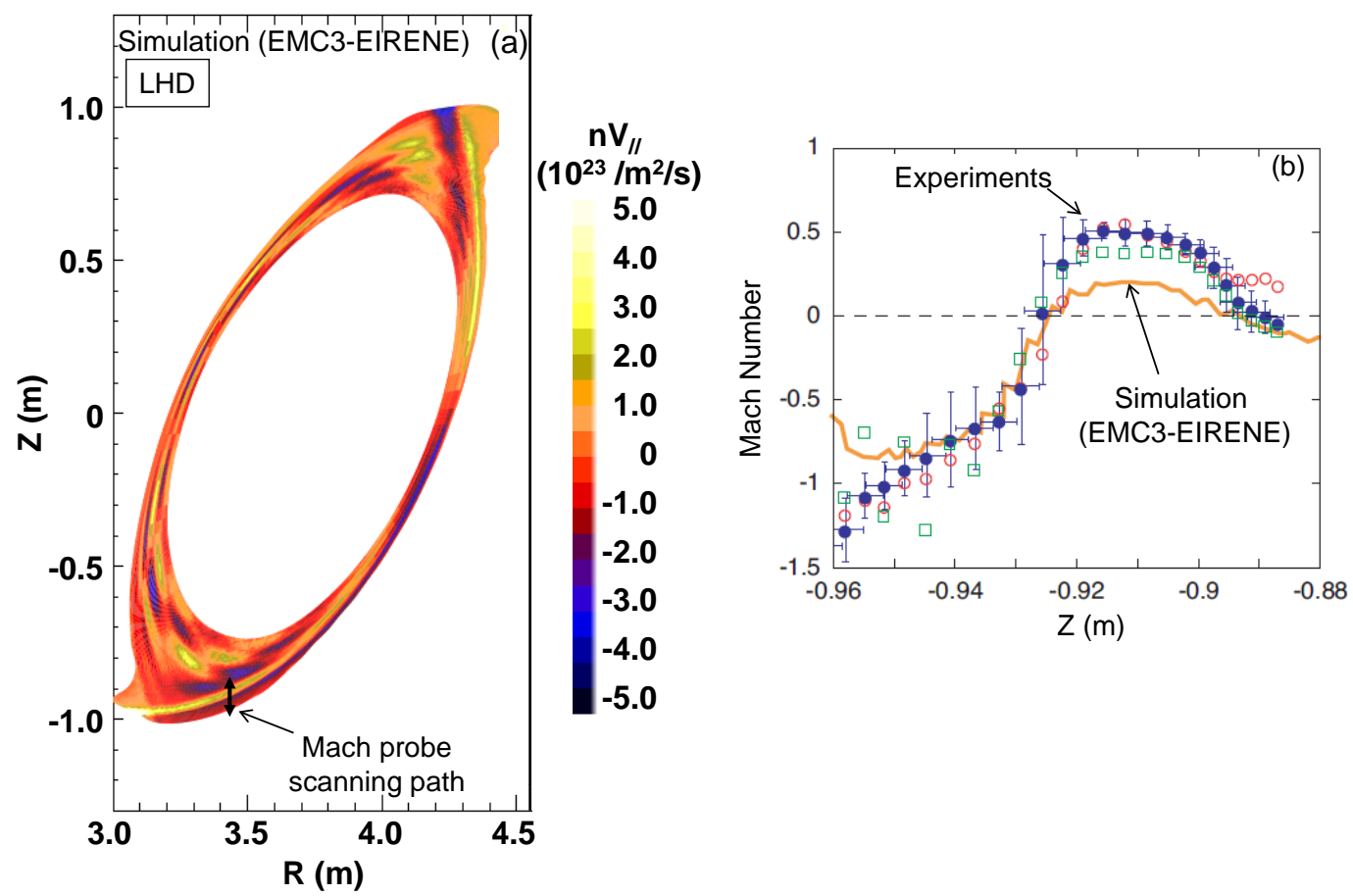

Fig.6 (a) 2D distribution of //-particle flux $\left(\mathrm{nV}_{/ /}\right)$in a poloidal cross section obtained by 3D simulation (EMC3-EIRENE). Bright colors (yellow and red) and dark colors (blue and black) represent //-flows in positive and negative toroidal directions, respectively. (b) Vertical profiles of Mach number measured by scanning Mach probe (symbols) together with the results of 3D simulations (solid line) [101]. The scanning path of the Mach probe is indicated in (a). 
Fig.7

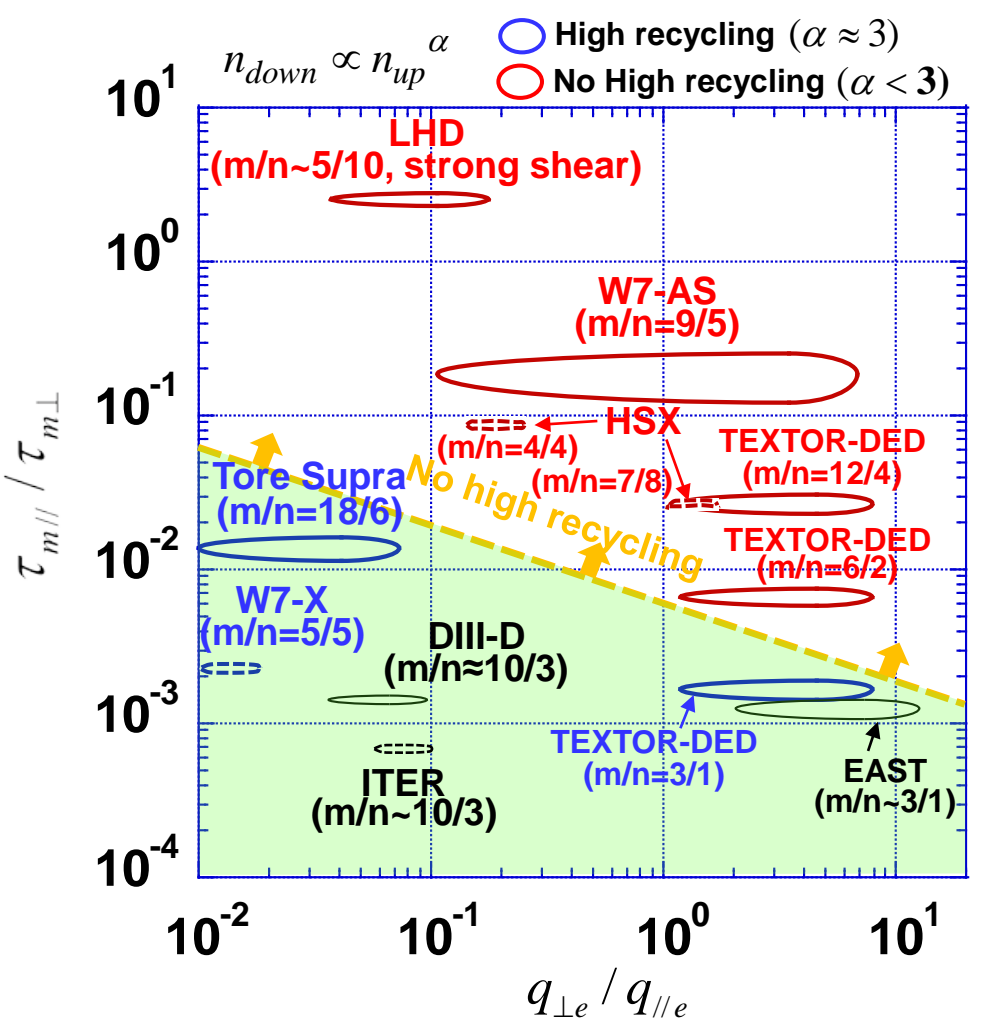

Fig. 7 Divertor density regime of various devices in terms of $\perp$ loss of //-momentum, $\tau_{m / /} / \tau_{m \perp}$, and replacement of //-energy flux with $\perp$-flux, $q_{\perp e} / q_{/ / e}$. Red: no high recycling regime $(\alpha<3)$, blue: with high recycling regime $(\alpha \approx 3)$, where $n_{\text {down }} \propto n_{\text {up }}{ }^{\alpha}$. The results from numerical simulations are plotted with dashed lines. The larger the $\tau_{m / /} / \tau_{m \perp}$ or $q_{\perp e} / q_{/ / e}$ are, the weaker the upstream and downstream coupling, i.e., density dependence, are. The shaded area indicates the operation domain given by $\left(\frac{\tau_{m / /}}{\tau_{m \perp}}\right)^{2}\left(\frac{q_{\perp e}}{q_{/ / e}}\right)<3.6 \times 10^{-5}$. 
Fig.8

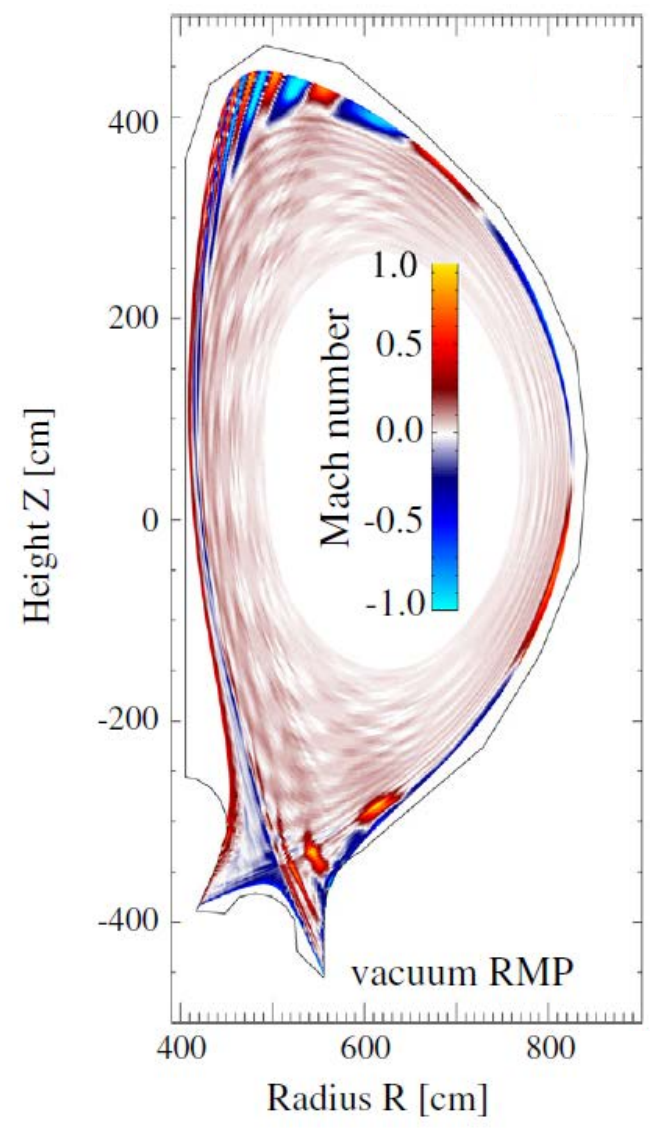

Fig.8 Mach number distribution in the ITER with MP application, obtained by 3D numerical simulation (EMC3-EIRENE) [29]. Red and blue colors represent //-flows in positive and negative toroidal directions, respectively. Counter streaming flows appear. 
Fig. 9
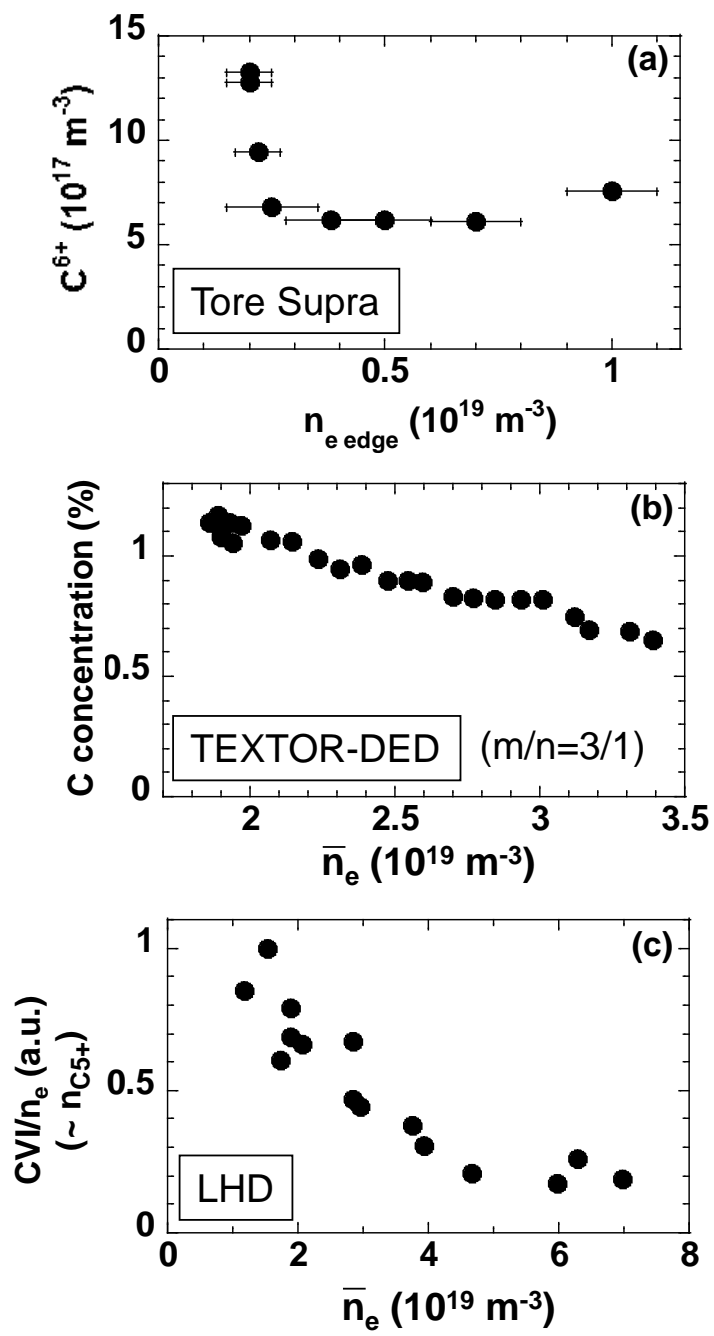

Fig. 9 Density dependence of carbon content in plasma for (a) Tore Supra [111], (b) TEXTOR-DED (m/n=3/1) [126] and (c) LHD. 
Fig.10

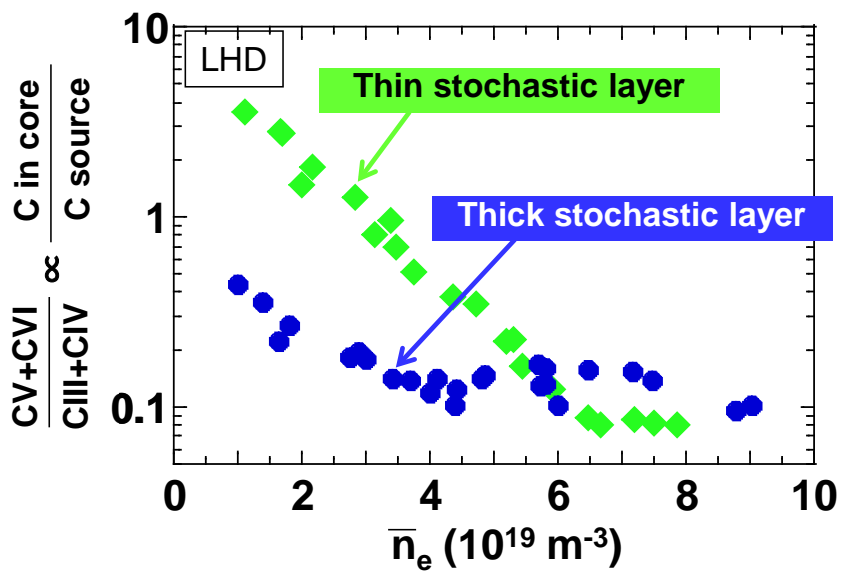

Fig.10 Density dependence of the carbon emission ratio (CV+CVI) to (CIII+CIV) for thin (diamonds) and thick (circles) stochastic layer widths in LHD [132]. The ratio is interpreted as the ratio of the carbon content in core plasma to the source, as discussed in the text. 
Fig. 11
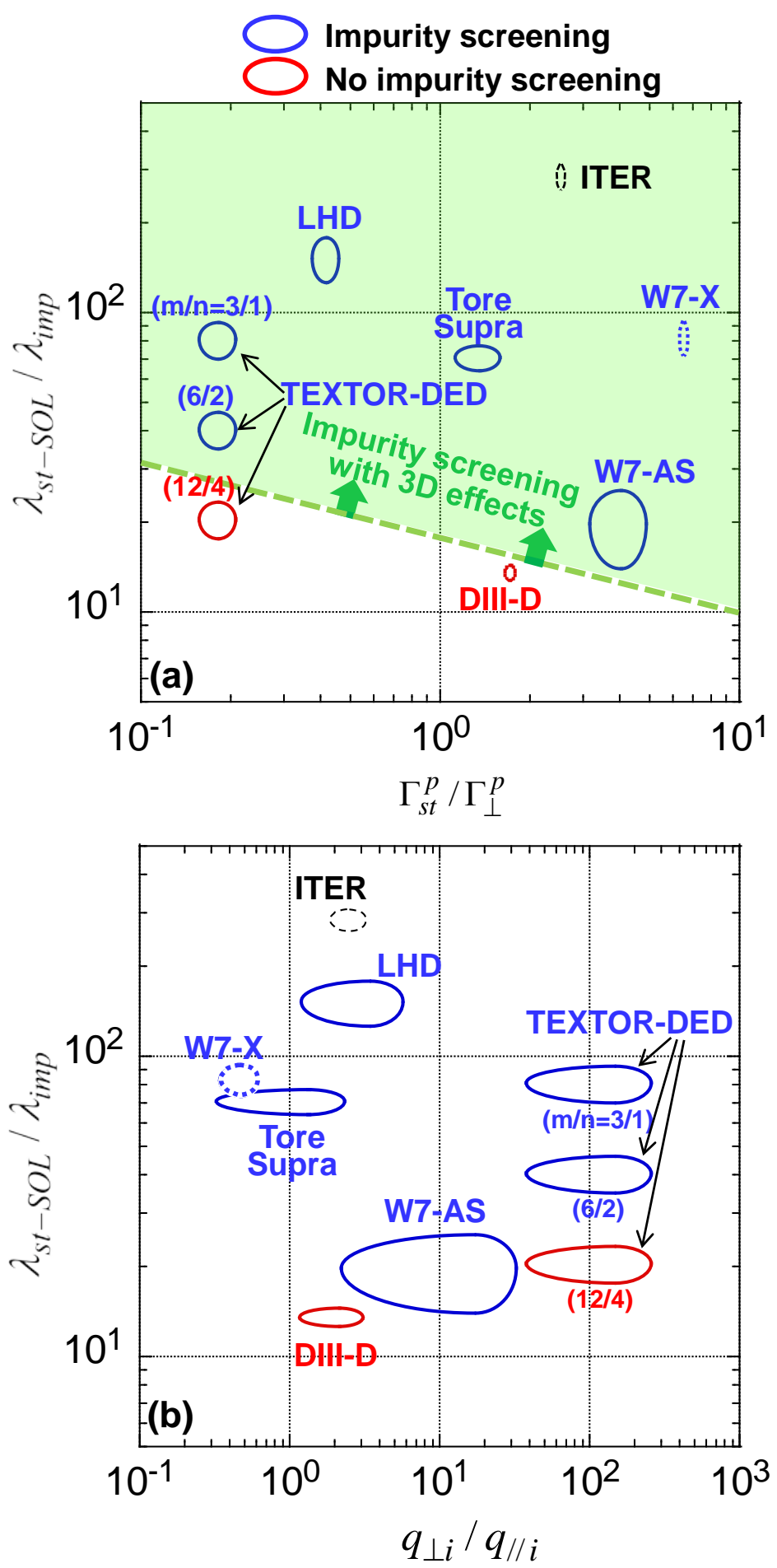
Fig.11 Operating domain of various devices in the space of (a) $\frac{\lambda_{s t-S O L}}{\lambda_{i m p}}$ and $\frac{\Gamma_{s t}^{p}}{\Gamma_{\perp}^{p}}$, and (b) $\frac{\lambda_{s t-S O L}}{\lambda_{\text {imp }}}$ and $\frac{q_{\perp i}}{q_{/ / i}}$. The observation of impurity screening is indicated with blue circles, and the observation of no impurity screening with red circles. The results from numerical simulations are plotted with dashed lines. $\frac{\lambda_{s t-S O L}}{\lambda_{\text {imp }}}$ is the ratio of the radial width of the stochastic layer or island divertor SOL to the neutral impurity penetration length for carbon. $\frac{\Gamma_{s t}^{p}}{\Gamma_{\perp}^{p}}$ and $\frac{q_{\perp i}}{q_{/ / i}}$ represent the enhancement of the radially outward particle flux and of $\perp$-ion energy flux, respectively. The operating domain of the upper half of the density range is plotted for all devices, taking into account the fact that the screening effects are pronounced always at high density operation. The results of numerical simulation are indicated with dashed lines. The shaded area in (a) indicates the operating domain given by $\left(\frac{\lambda_{s t-S O L}}{\lambda_{\text {imp }}}\right)\left(\frac{\Gamma_{s t}^{p}}{\Gamma_{\perp}^{p}}\right)^{1 / 4}>18$. 
Fig. 12

\section{W7-AS}

Stable detach. Unstable detach. with Large $\Delta \mathrm{X}_{\text {LCFS-div }}$ with small $\Delta \mathrm{X}_{\text {LCFS-div }}$

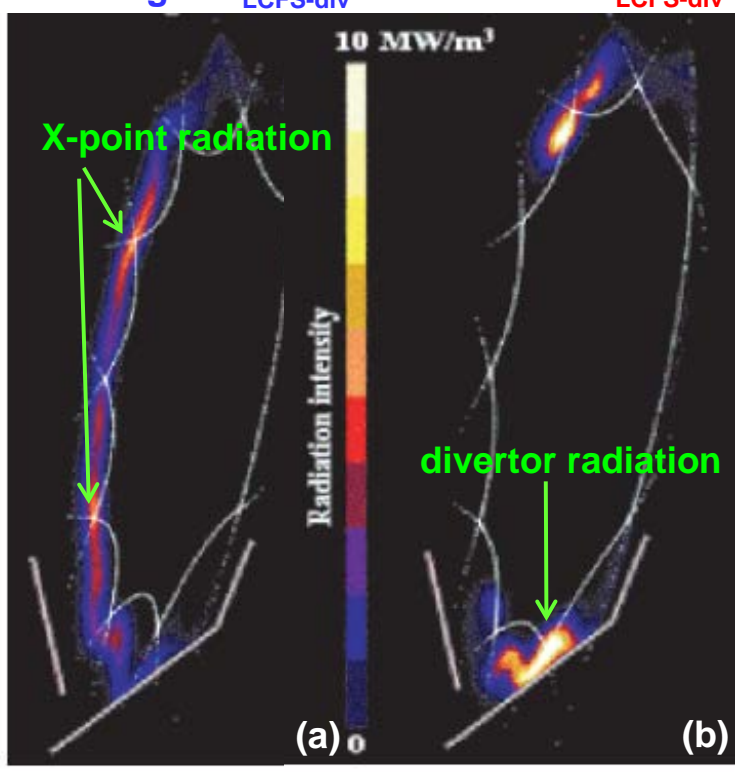

Carbon radiation distribution (EMC3-EIRENE)

Fig.12 Carbon radiation distribution in W7-AS for (a) stable detachment with large $\Delta x_{L C F S-d i v}$ and for (b) unstable detachment with small $\Delta x_{L C F S-d i v}$, obtained by numerical simulations (EMC3-EIRENE). [156] 
Fig. 13
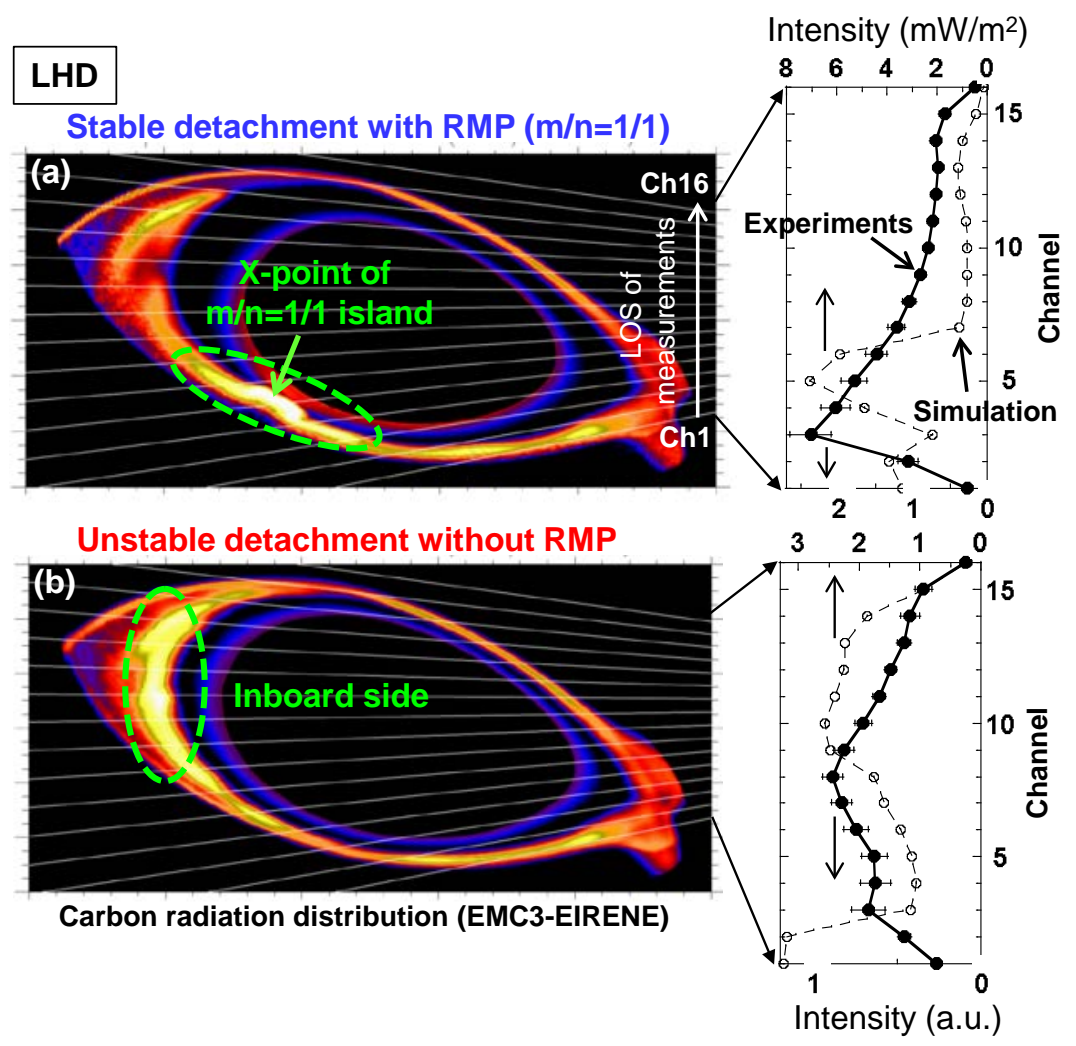

Fig.13 Carbon radiation distribution in LHD for (a) stable detachment with MP ( $\mathrm{m} / \mathrm{n}=1 / 1)$ application and for (b) unstable detachment without MP, obtained by numerical simulations (EMC3-EIRENE). The line integrated radiation profile measurements along the line of sight as indicated with white lines, are plotted together on the right column, in comparison with the simulations [159]. 
Fig. 14
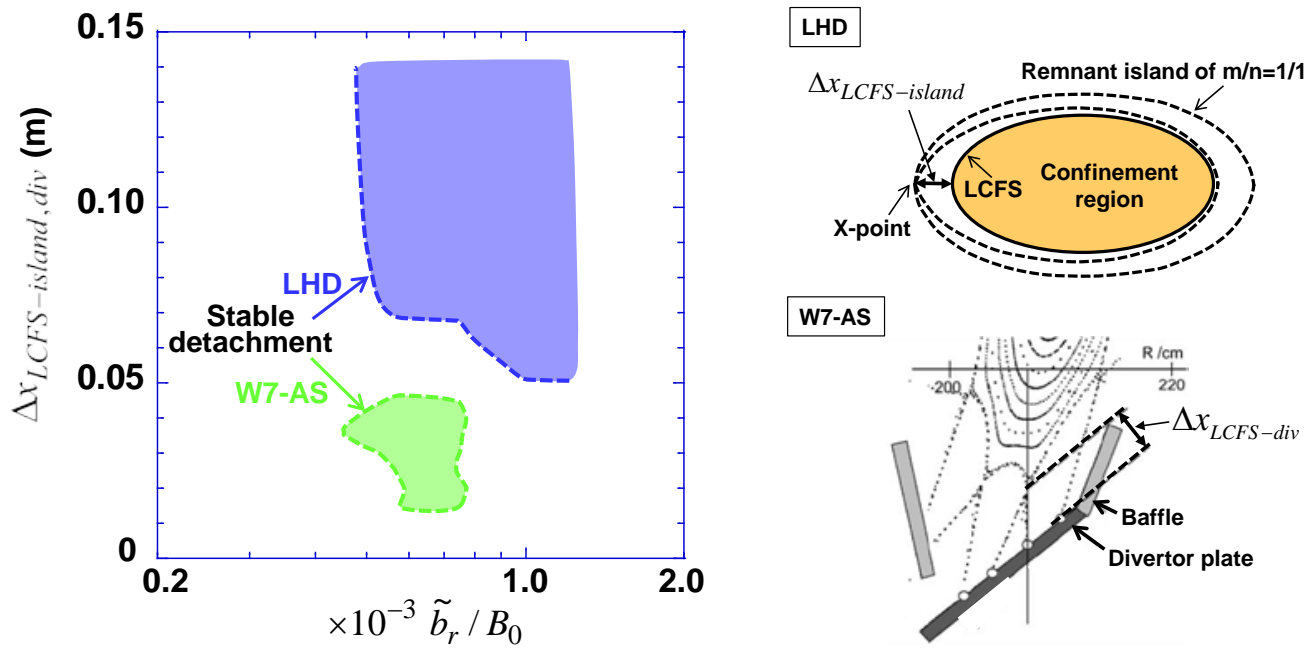

Fig. 14 Operation domain of the stable detachment in LHD and W7-AS in terms of $\Delta x_{\text {LCFS-island }}\left(\Delta x_{L C F S-d i v}\right)$ and $B_{r} / B_{t}$. The definitions of $\Delta x_{L C F S-i s l a n d}$ and $\Delta x_{L C F S-d i v}$ are given in the right figures. 
Fig.15

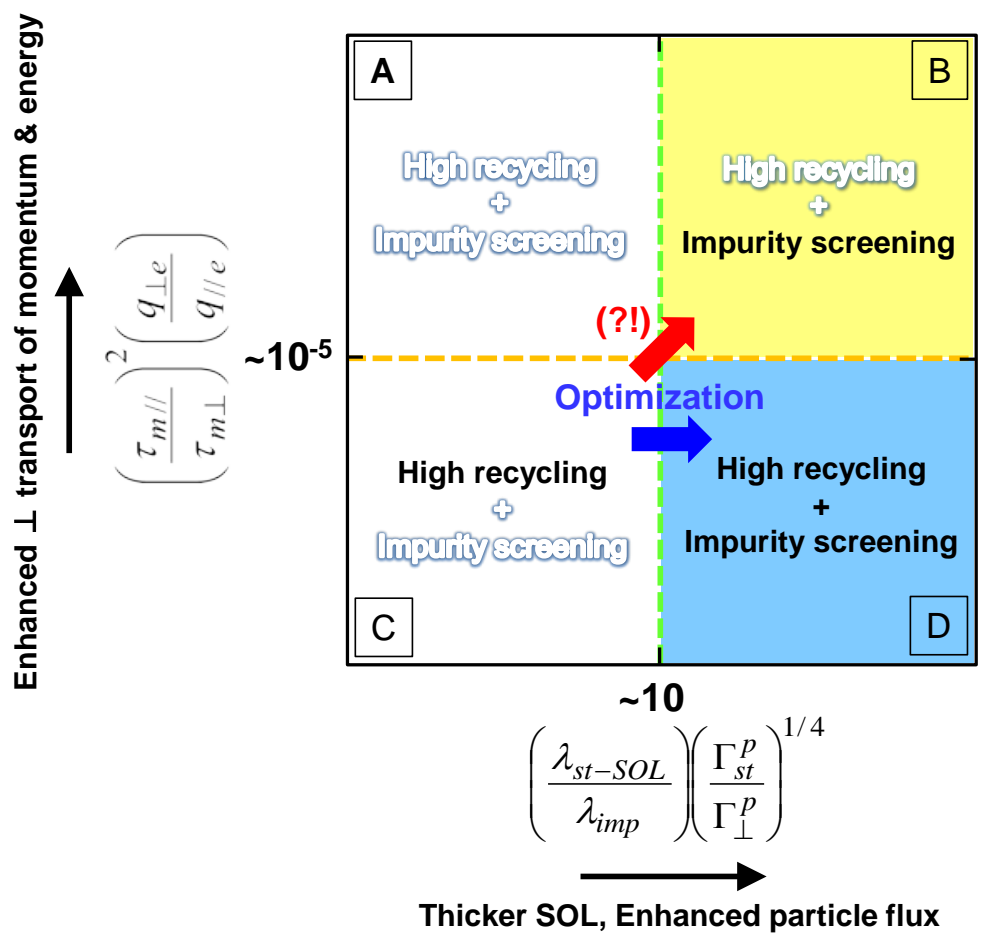

Fig.15 Schematic of possible optimization of 3D divertor configuration in terms of $\left(\frac{\tau_{m / /}}{\tau_{m \perp}}\right)^{2}\left(\frac{q_{\perp e}}{q_{/ / e}}\right)$ (as a measure of enhancement of $\perp$-energy and momentum transport) and of $\left(\frac{\lambda_{s t-S O L}}{\lambda_{\text {imp }}}\right)\left(\frac{\Gamma_{s t}^{p}}{\Gamma_{\perp}^{p}}\right)^{1 / 4}$ (as a measure of SOL thickness and of enhanced outward particle flux). The operation space is divided into four domains (A to D) according to the operation boundaries of the high recycling regime and of impurity screening. 


\section{APPENDIX}

Fig.A1
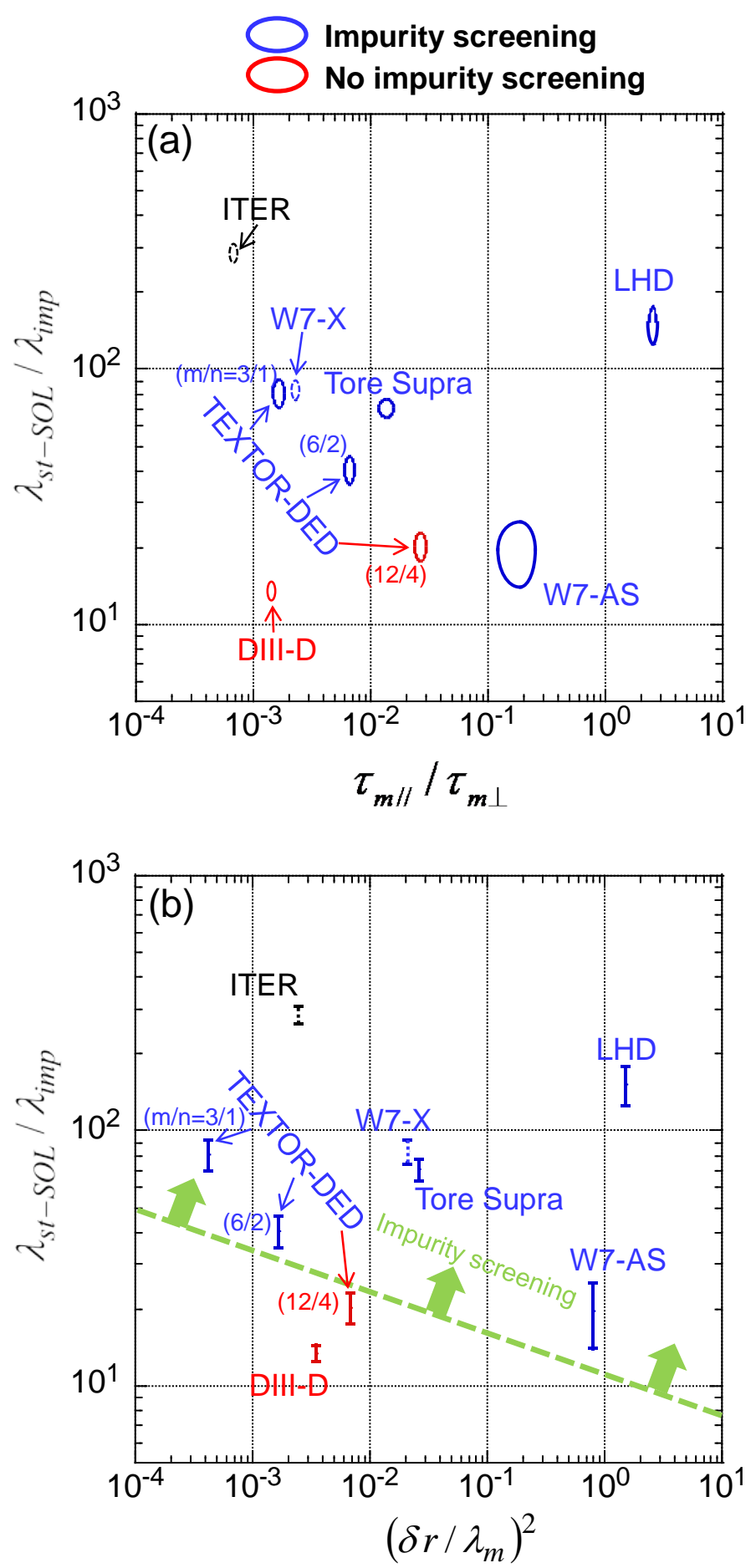

Fig.A1 Operating domain of each device in the space of (a) $\tau_{m / /} / \tau_{m \perp}-\lambda_{s t-S O L} / \lambda_{i m p}$ and (b) $\left(\delta r / \lambda_{m}\right)^{2}-\lambda_{\text {st-SOL }} / \lambda_{\text {imp }}$. 